\title{
Deep inelastic scattering at strong coupling from gauge/string duality : the saturation line
}

\section{Y. Hatta and E. Iancu}

Service de Physique Théorique, CEA Saclay, F-91191 Gif-sur-Yvette, France

E-mail: Yoshitaka.Hatta@cea.fr, Edmond.Iancu@cea.fr

\section{A. H. Mueller \\ Department of Physics, Columbia University, New York, NY 10027, USA \\ E-mail: amh@phys.columbia.edu}

\begin{abstract}
For gauge theories which admit a dual string description, we analyze deep inelastic scattering at strong 't Hooft coupling and high energy, in the vicinity of the unitarity limit. We discuss the onset of unitarity corrections and determine the saturation line which separates weak scattering from strong scattering in the parameter space of rapidity and photon virtuality. We discover that the approach towards unitarity proceeds through two different mechanisms, depending upon the photon virtuality $Q^{2}$ : single Pomeron exchange at relatively low $Q^{2}$ and, respectively, multiple graviton exchanges at higher $Q^{2}$. This implies that the total cross-section at high energy and large $Q^{2}$ is dominated by diffractive processes. This is furthermore suggestive of a partonic description where all the partons have transverse momenta below the saturation momentum and occupation numbers of order one.
\end{abstract}




\section{Contents}

1. Introduction 1

2. Saturation momentum and geometric scaling in perturbative QCD

3. DIS at strong coupling 13

3.1 The setup 13

3.2 Limit of the single Pomeron approximation : A lesson from a sum rule 17

甘. Mapping the high-energy 'phase diagram' 22

4.1 Anomalous dimension of twist-two operators in $\mathcal{N}=4 \mathrm{SYM}$

4.2 The Pomeron propagator 24

4.3 Saturation line from multiple graviton exchanges 29

5. Physical discussion: Towards a partonic picture at strong coupling 33

A. On the breakdown of the eikonal approximation at ultrahigh energies 37

\section{Introduction}

High energy, small angle, scattering has always been the subject of much attention, since the early days of elementary particle physics. While the phenomenological Regge theory, centered around the 'soft Pomeron', has met with considerable success in reproducing the experimental data for hadronic collisions, a fundamental understanding of this theory rooted in QCD is still lacking and seems to even transcend our present capabilities - as neither perturbative QCD, nor lattice gauge theory, can be applied in the relevant 'Regge kinematics' (high energy, but soft momentum transfer).

In perturbative QCD, the resummation of the logarithmically enhanced diagrams leads to the BFKL, or 'hard', Pomeron [1] which predicts a power-like growth with $s$ for the gluon occupation number and the scattering amplitudes. The growth is however faster than observed in experiments, and is also problematic from a conceptual point of view as it violates unitarity. Within the past decade there has been considerable activity and some important progress towards understanding the unitarization of the BFKL Pomeron within pQCD. These efforts have been mainly oriented in two directions: (i) the calculation [2] and proper implementation [3] of the next-to-leading order corrections to the linear BFKL equation, and (ii) the inclusion of the non-linear effects, like multiple scattering and gluon saturation, responsible for the unitarization of the scattering amplitudes [4, 5, 6, 0, 8, 9].

Whereas weak coupling techniques are undoubtedly useful for the high-energy problems which involve, at least, one hard momentum scale — so like deep inelastic scattering at small $x$ 
and large photon virtuality $Q^{2}\left(s \gg Q^{2} \gg \Lambda_{\mathrm{QCD}}^{2}\right)$, or hadronic scattering with large momentum transfer $\left(s \gg|t| \gg \Lambda_{\mathrm{QCD}}^{2}\right)$ - on the other hand it remains unclear whether such approaches can shed any light on the 'soft' $\left(|t| \lesssim \Lambda_{\mathrm{QCD}}^{2}\right)$ aspects of Regge phenomenology. For instance, is there any relation between the soft and the hard Pomerons, and if so, then how to describe the transition between the two ? Such questions are particularly difficult because of our inability to explore the transitive region where the QCD coupling constant becomes strong. It is therefore interesting to study model field theories which are analytically accessible at strong coupling and which share some of the basic features of QCD at high energy - like the existence of a partonic description at weak coupling and a Regge-like behavior at high energy, for any value of the coupling.

$\mathcal{N}=4$ supersymmetric Yang-Mills (SYM) theory with 'color' gauge group $\mathrm{SU}\left(N_{c}\right)$ and the 't Hooft coupling constant $\lambda=g^{2} N_{c}$ treated as a free parameter offers a particularly interesting theoretical laboratory for this purpose. At weak coupling, the high energy behavior is just like in perturbative QCD - in particular, to lowest order, the BFKL equation is formally identical in QCD and $\mathcal{N}=4$ SYM 10 — while at strong coupling and in the large $N_{c}$ limit the AdS/CFT correspondence 11] maps the theory onto a weakly coupled superstring theory which naturally exhibits Regge behavior (see, e.g., [12, 13, 14, 15, 16]). Such a change of behavior with increasing the coupling strength bears some resemblance to the transition from the hard Pomeron to the soft Pomeron in QCD, although some important differences persists. (For instance, when moving from weak to strong coupling, the Pomeron intercept decreases in QCD, while it increases in the $\mathcal{N}=4$ SYM theory.) Thus one can hope that some useful insights for the corresponding QCD problems may be gleaned from a study of the Regge regime in $\mathcal{N}=4 \mathrm{SYM}$ theory and its variants.

In this paper we study deep inelastic scattering (DIS) in strongly coupled $\mathcal{N}=4$ theory at small- $x$ where $x \simeq Q^{2} / s$ is the usual Bjorken variable. The basic formulation was given by Polchinski and Strassler [15] in the strict large $-N_{c}$ limit $\left(N_{c} \rightarrow \infty\right.$ at fixed energy), where however no unitarity issue arises: being suppressed by a factor $1 / N_{c}^{2}$, the elementary scattering amplitude is always smaller than one, and is dominated by the exchange of a single 'Pomeron'. In this strong-coupling context, the 'Pomeron' is the $t$-channel object exchanged at tree-level and at high energy in the dual string theory [16] — a reggeized graviton propagating in the curved space-time that the string theory lives in and which is asymptotically $A d S_{5} \times S^{5}$.

Thus, in order to study unitarization, one should rather consider the high-energy limit $s \rightarrow \infty$ at large, but fixed, values of $N_{c}$, and this is what we shall do in this paper. This is again similar to QCD (at large $N_{c}$ ), where the unitarity corrections are suppressed by inverse powers of $N_{c}$, hence a flexible definition of the 'large $-N_{c}$ limit' is necessary to observe such phenomena. The problem of unitarization in the context of string theory is notoriously difficult, even at weak (string) coupling $g_{s}^{2} \propto 1 / N_{c}^{2}$, because of the need to resum multi-loop string amplitudes to all orders, and here we shall not attempt to provide a full analysis in that sense (see however the approaches in Refs. [12, 17, 18]). Rather, our goal is more modest and also more pragmatic: Following again the example of weakly-coupled QCD - where it has been shown [19, 20, 21, 22] that one can follow the onset of unitarity corrections via an appropriate extrapolation of the results at weak scattering (in that case, the single BFKL-Pomeron exchange) -, we shall compute, at strong coupling, the saturation line which separates the weak-scattering from the 
strong-scattering regions in the kinematical plane $\left(x, Q^{2}\right)$ for DIS.

Specifically, while staying in the weak-scattering regime — which at strong coupling, like at weak coupling, corresponds to sufficiently large values of $Q^{2}$ for a given value $x \ll 1-$, we shall first identify those contributions to the scattering amplitude which become dominant when extrapolating towards the unitarity limit, and then deduce the saturation line as the curve $Q_{s}^{2}(x)$ along which the amplitude is constant and of $\mathcal{O}(1)$. What at a first sight may look as a rather straightforward exercise in unitarizing the single-Pomeron exchange of Refs. [15, 16], turns out to be quite subtle, because of several issues:

First, the formulation of the unitarity constraint is rather non-trivial in the context of DIS, where the scattering is initiated by an off-shell, electromagnetic, current (actually, an $\mathcal{R}$-current in $\mathcal{N}=4 \mathrm{SYM}$ ). Within the context of pQCD, this issue is most conveniently solved by using the dipole factorization of DIS at small $x$ [24, 25], where one can unambiguously identify the on-shell hadronic state which scatters off the proton, and for which the unitarity bound can be properly formulated: the quark-antiquark excitation of the virtual photon, or 'color dipole' (see also Sect. 2 below, and Refs. [26, 27] for more detailed discussions). It turns out that a formally similar factorization holds at strong coupling as well, with the color dipole replaced by a vector-field fluctuation of the background metric (the ' $\mathcal{R}$-boson'), which propagates onshell before eventually scattering off the 'dilaton' target 15. This makes it natural to enforce the unitarity constraint at the level of the elementary scattering between the $\mathcal{R}$-boson and the dilaton. This constraint will determine our saturation line.

Second, unlike what happens in pQCD, where the single (BFKL) Pomeron exchange controls the dipole amplitude in the vicinity of the unitarity limit for all the relevant values of $x$ and $Q^{2}$, the situation at strong coupling appears to be more complex, and also more interesting: there, the single-Pomeron exchange represents the dominant contribution (when approaching the saturation line, once again) only for sufficiently low values of $Q^{2}$, up to a critical value $Q_{c}^{2} \propto \exp \left\{4 \ln N_{c}^{2} / \sqrt{\lambda}\right\}$. But for $Q^{2}>Q_{c}^{2}$, a new mechanism takes up the leading role, namely the diffractive scattering via the exchange of two, or more, elementary (massless) gravitons. Note that $Q_{c}^{2} \rightarrow \infty$ in the strict large $-N_{c}$ limit, which explains why this new mechanism has not been included in the analysis in Ref. [15]. Interestingly, the picture of DIS at high energy and $Q^{2}>Q_{c}^{2}$ that we shall arrive is quite close to that of Regge scattering in superstring theory in flat space, as studied by Amati, Ciafaloni and Veneziano [12].

In fact, for a strongly-coupled gauge theory having a dual string description, the graviton exchange is naturally more effective, at high energy and large $Q^{2}$, than the single-Pomeron one: the amplitude for one-graviton exchange grows very fast with the energy, as $s \sim 1 / x$, because the graviton has spin $j=2$, and, moreover, its long-range non-locality can efficiently match the large separation in scales between the highly virtual $\mathcal{R}$-current and the dilaton. (Of course, being real, the single graviton exchange cannot contribute to the DIS structure functions, but multiple exchanges can do so, via diffractive final states where the outgoing fields are separated in rapidity.) The reason why the single-Pomeron exchange is nevertheless found to dominate at relatively low $Q^{2}$ is because, in the $A d S_{5}$ geometry, the massless graviton is turned off at very short separations (in the radial direction of $A d S_{5}$ ), by the curvature of space-time. Or, by the holographic principle of AdS/CFT (which becomes manifest in the factorization of DIS alluded to above), small separations in $A d S_{5}$ correspond to low virtualities $Q^{2}$ on the gauge theory side. 
This transition, from a single-Pomeron to multiple graviton exchanges, with increasing $Q^{2}$ has important consequences on the shape of the saturation line, correspondingly obtained as the juxtaposition of two curves which smoothly match with each other at $Q^{2}=Q_{c}^{2}$, and also on the behaviour of the DIS cross-section in the vicinity of this line. We thus find that, at large $Q^{2}>Q_{c}^{2}$, the structure function $F_{2}\left(x, Q^{2}\right)$ is strongly peaked at the saturation momentum - namely, $F_{2} \propto N_{c}^{2} Q^{2}$ when $Q^{2}<Q_{s}^{2}(x)$, but $F_{2} \propto 1 /\left(N_{c}^{2} Q^{2}\right)$ when $Q^{2} \gg Q_{s}^{2}(x)$-, which is suggestive of a partonic interpretation with all the partons living at transverse momenta $k_{\perp} \lesssim Q_{s}(x)$ and having occupation numbers of $\mathcal{O}(1)$. This is further supported by an analysis of the 'sum-rule' $\int \mathrm{d} x F_{2}\left(x, Q^{2}\right)$ which expresses the energy-momentum conservation: at large $Q^{2}$, this sum-rule appears to be saturated by values of $x$ near the saturation line, i.e., the largest values of $x$ at which one can still find the partons. Needless to say, at strong coupling such a partonic language must be used with care, since the standard relations between structure functions and parton distributions hold, strictly speaking, only in perturbation theory. It is nevertheless interesting that the picture of parton saturation suggested by our analysis appears as a natural continuation towards strong coupling of the corresponding results as weak coupling, as obtained in pQCD 19, 28, 29, 4, 5, 6, 7, 8, 9, 27.

This paper is organized as follows: In Section 2, we give a self-contained review of highenergy DIS in QCD at weak coupling, in the framework of dipole factorization. The focus is on the definition and the construction of the saturation line, with the purpose of facilitating the subsequent discussion at strong coupling, and also the comparison between the weak and, respectively, strong-coupling scenarios. In Section 3, we describe our setup of DIS at strong coupling following [15] and demonstrate a limitation of the single-Pomeron exchange approximation, which fails to saturate the energy-momentum sum rule at large $Q^{2}$. This points out towards the importance of the massless graviton exchanges, which are then discussed at length in Section 4. On this occasion, we use and extend the 'Pomeron' approach initiated in Refs. [14, 15] and more systematically developed in [16], with the purpose of demonstrating the reemergence of the massless graviton propagator at large $Q^{2}$. We then describe multiple graviton exchanges at a heuristic level, using in particular the results in Refs. [12, 17, 18] to deduce the onset of unitarity corrections. We summarize our results for the saturation line in a 'phase diagram', Fig. 3, for gauge theories at high energy and strong 't Hooft coupling, to be compared with the corresponding diagram at weak coupling in Fig. 1. Section 5 is devoted to a discussion of our results, in relation with a possible partonic interpretation.

\section{Saturation momentum and geometric scaling in perturbative QCD}

As mentioned in the Introduction, deep inelastic lepton-hadron scattering (DIS) at relatively high virtuality $Q^{2} \gg \Lambda_{\mathrm{QCD}}^{2}$ for the exchanged photon and in the high-energy limit $s \gg Q^{2}$ represents a rather clean laboratory for a theoretical study of unitarization in the framework of perturbative QCD - in particular, for the calculation of the saturation momentum. More precisely, in what follows we shall work in the 'small- $x$ ' regime of DIS, characterized by

$$
\alpha_{s} \ll 1, \quad x \equiv \frac{Q^{2}}{s} \ll 1, \quad \text { and } \quad \alpha_{s} \ln \frac{1}{x} \geq 1 .
$$

A 'lowest-order calculation' in this regime requires an all-order resummation of the perturbative contributions of order $\left(\alpha_{s} \ln (1 / x)\right)^{n}$, with $n \geq 1$. This resummation can be performed by 
solving appropriate evolution equations, constructed within pQCD, which are generally nonlinear, with the non-linear terms accounting for the 'unitarity corrections' - gluon saturation and multiple scattering [4, 5, 7, 8, 30]. However, a linear approximation - the BFKL equation 11, 25, 2] - holds within an intermediate range of energies, in which the non-linear effects remain parametrically small. The boundary of the validity region for this approximation in the kinematical plane $\left(x, Q^{2}\right)$ is known as the 'saturation line'; this is the curve $Q^{2}=Q_{s}^{2}(x)$ along which the scattering amplitude is constant and of order one. Remarkably, it turns out that the position of this line can be determined via calculations based on the BFKL approximation alone, without a detailed knowledge of the non-linear dynamics responsible for unitarization [20, 21, 22]. In what follows, we shall briefly review this calculation to 'leading-logarithmic accuracy' (LLA), i.e., at the level of the leading-order BFKL equation. (See also Ref. [31, 32] for more accurate analyses, including NLO effects.)

To LLA, the cross-sections in QCD at high energy can be computed within a factorization scheme known as ' $k_{T}$-factorization' (see [33, 26]), which is consistent with the non-locality of the high-energy evolution in transverse coordinates. When applied to DIS, this is most suggestively written as dipole factorization : the virtual photon $\gamma^{*}$ fluctuates into a quark-antiquark pair in a color-singlet state, or 'color dipole', which then scatters off the gluon field inside the target. More precisely, the DIS structure function $F_{2}$ is computed as

$$
F_{2}\left(x, Q^{2}\right)=\frac{Q^{2}}{4 \pi^{2} \alpha_{\mathrm{em}}} \sigma_{\gamma^{*} p}\left(Q^{2}, x\right)
$$

with $\sigma_{\gamma^{*} p}$ the total $\gamma^{*} p$ cross-section :

$$
\sigma_{\gamma^{*} p}\left(Q^{2}, x\right)=\int_{0}^{1} \mathrm{~d} z \int \mathrm{d}^{2} \boldsymbol{r} \sum_{a=T, L}\left|\Psi_{a}(z, r)\right|^{2} \sigma_{\text {dipole }}(r, x),
$$

where the subscript $a$ refers to the polarization of the virtual photon (transverse or longitudinal), and $\Psi_{a}(z, r)$ is the wavefunction describing the dissociation of the virtual photon into a $q \bar{q}$ pair with transverse size $r=|\boldsymbol{r}|$ and where the quark (antiquark) takes away a fraction $z$ (respectively, $1-z$ ) of the photon energy. For massless quarks, one finds [24]

$$
\begin{aligned}
\left|\Psi_{T}(z, r)\right|^{2} & =\mathcal{F}\left[z^{2}+(1-z)^{2}\right] \bar{Q}^{2} K_{1}^{2}(\bar{Q} r), \\
\left|\Psi_{L}(z, r)\right|^{2} & =4 \mathcal{F} Q^{2} z^{2}(1-z)^{2} K_{0}^{2}(\bar{Q} r),
\end{aligned}
$$

where $\bar{Q}^{2} \equiv z(1-z) Q^{2}, K_{0}$ and $K_{1}$ are modified Bessel functions, and $\mathcal{F} \equiv\left(N_{c} \alpha_{\mathrm{em}} / 2 \pi^{2}\right) \sum_{f} e_{f}^{2}$, with $e_{f}$ the electric charge of the quark with flavor $f$. The factor of $N_{c}$ comes from the sum over the color degrees of freedom of the quarks. The integration over $r$ in Eq. (2.3) is effectively restricted to $r<1 / \bar{Q}$ by the Bessel functions, which are exponentially suppressed for $\bar{Q} r \gg 1$.

Furthermore, Eq. (2.3) involves the total cross-section $\sigma_{\text {dipole }}(x, r)$ for the scattering between a color dipole with transverse size $r$ and the hadronic target. For the present purposes, it is convenient to take the target itself as a collection of dipoles, succinctly referred to as the onium. In its own rest frame, the onium is a collection of dipoles with sizes $r^{\prime} \leq R_{0}$ distributed according to a density function $n\left(r^{\prime}\right)$ and which are homogeneously distributed in impact parameter space within a disk of radius $R_{0}$. Note that $R_{0}$ plays the role of an 'infrared cutoff' for this dipole distribution, in the sense that this is the maximally allowed dipole size. To ensure applicability 
of perturbation theory, we shall assume that $1 / R_{0}^{2} \gg \Lambda_{\mathrm{QCD}}^{2}$ (but this assumption is often relaxed in phenomenological studies of DIS, in which case $R_{0}$ is identified with the proton radius). Also, it is convenient to choose $Q^{2} \gg 1 / R_{0}^{2}$, in which case the integral in Eq. (2.3) is dominated by relatively small dipole sizes $r$ (for the projectile dipole), such that $r \ll R_{0}$.

To the accuracy of interest, the dipole-onium cross-section can be evaluated as

$$
\sigma_{\text {dipole }}(r, \tau)=\int_{0}^{\infty} \frac{\mathrm{d} r^{\prime 2}}{r^{\prime 2}} \sigma_{\mathrm{DD}}\left(r, r^{\prime}, \tau\right) n\left(r^{\prime}\right)
$$

where $\tau \equiv \ln (1 / x)$ is the 'rapidity' and $\sigma_{\mathrm{DD}}\left(r, r^{\prime}, \tau\right)$ is the total cross-section for the scattering between two dipoles with transverse sizes $r$ and $r^{\prime}$ separated by a rapidity interval $\tau$. The dipole density is chosen as

$$
n\left(r^{\prime}\right)=\left(\frac{r^{\prime 2}}{R_{0}^{2}}\right)^{\Delta} \Theta\left(R_{0}-r^{\prime}\right),
$$

where the power $\Delta$ plays the role of an 'anomalous dimension': indeed, the standard situation in perturbative QCD (at least for sufficiently small $r^{\prime}$ ) is $\Delta=0$, corresponding to the bremsstrahlung of small dipoles. The associated distribution is not normalizable (it exhibits a logarithmic divergence at $r^{\prime} \rightarrow 0$ ), but this poses no problem for the calculation of the crosssection (2.5) since $\sigma_{\mathrm{DD}}\left(r, r^{\prime}, \tau\right)$ vanishes sufficiently fast when $r^{\prime} \rightarrow 0$ (see below). A normalizable hadronic state would have $\Delta>0$, in which case $n\left(r^{\prime}\right)$, once properly normalized, would have the interpretation of the probability density to find a dipole of size $r^{\prime}$ in the target. In what follows, we shall consider the cases where $\Delta$ is positive or zero.

We shall soon compute the cross-section (2.5) in the BFKL approximation and then use the result, in association with the unitarity constraint, to deduce an equation for the saturation line. In preparation of this, it is useful to open a parenthesis and explain our specific use of the unitarity constraint for the present purposes. (A similar use will be made in the subsequent sections in the context of DIS at strong coupling.)

The unitarity bound is most easily formulated in terms of the scattering amplitude $T(r, b, \tau)$ at fixed impact parameter $b$. It then reads $T(r, b, \tau) \leq 1$, where the upper bound $T=1$ (the 'black disk limit') describes a situation where the scattering occurs with probability one. Note that we use conventions in which the $S$-matrix is written as $S=1-T$, with $T$ a real quantity at high energy. (That is, our $T$ corresponds to the imaginary part of the conventionally-defined 'scattering amplitude', which becomes predominantly imaginary at high energy.) However, the previous formulæ, Eqs. (2.3)-(2.5), involve directly the cross-sections, which are obtained from the respective amplitudes after integrating over $b: \sigma_{\text {dipole }}(r, \tau)=2 \int \mathrm{d}^{2} b T(r, b, \tau)$, etc. The unitarity constraint then becomes more subtle: with increasing energy, a cross-section can rise indefinitely, even after the 'black disk' limit has been achieved at central impact parameters, because of the radial expansion of the black disk. This expansion is however quite slow, logarithmic in the energy (to comply with Froissart bound), which is indeed much slower than the corresponding power-like increase of the amplitude in the BFKL regime (see below). Hence, it is possible to neglect the Froissart expansion (or, at least, treat this in an adiabatic approximation) in a first study of unitarization. Then, the unitarity bound reads $\sigma_{\mathrm{DD}}\left(r, r^{\prime}, \tau\right) \leq 2 \pi r_{>}^{2}$ (with $r_{>}=\max \left(r, r^{\prime}\right)$ ) for the dipole-dipole scattering, and $\sigma_{\text {dipole }}(x, r) \leq 2 \pi R_{0}^{2}$ for the dipoleonium one. It is in fact more suggestive to introduce scattering amplitudes averaged over $b$, e.g., $T(r, \tau) \equiv \sigma_{\text {dipole }}(r, \tau) /\left(2 \pi R_{0}^{2}\right)$, in terms of which the unitarity bound reads simply $T \leq 1$. 
We can now explain our strategy for computing the saturation line: Starting in the regime where the scattering is weak, $T \ll 1$, we compute the (average) scattering amplitude from the solution to the BFKL equation and then extrapolate the result towards the unitarity limit $T \sim \mathcal{O}(1)$. The saturation momentum $Q_{s}(\tau)$ is then obtained via the condition

$$
T(r, \tau) \simeq 1 \quad \text { for } \quad r \simeq 1 / Q_{s}(\tau) .
$$

That is, $1 / Q_{s}(\tau)$ is the critical dipole size for the onset of unitarity corrections (in the form of multiple scattering) in dipole-target scattering at rapidity $\tau$ [20, 21]. This is also the critical momentum scale for parton saturation in the target wavefunction [19, 28, 29, 6, 9], in a frame where the target has rapidity $\tau$ : for transverse momenta $k_{\perp} \lesssim Q_{s}(\tau)$, the gluon occupation numbers saturate at a value $n_{g} \sim 1 /\left(\alpha_{s} N_{c}\right)$, which in turn implies that the (anti)quark occupation numbers saturate at a value $n_{q} \sim 1$. (Recall that, at small $x$ and relatively low $k_{\perp}$, the quark distribution is driven by gluons, which dominate the small- $x$ part of the wavefunction.) This profound link between dipole unitarization and gluon saturation in pQCD can be understood as a consequence of the relation $T(\tau, r) \sim \bar{\alpha}_{s} n_{g}\left(\tau, k_{\perp} \sim 1 / r\right)$, valid in the BFKL regime, between the dipole amplitude and the gluon occupation number.

By using Eq. (2.7) together with the BFKL solutions to be shortly presented, we shall compute the saturation lines for both dipole-dipole, and dipole-onium, scattering. For more clarity, let us anticipate here the main conclusions that we shall arrive at: (i) The value of the saturation momentum depends upon the nature of the target, but its evolution with increasing energy does not - the evolution is universal. (ii) The saturation momentum of the onium coincides with that of its 'softest' component — the constituent dipole with maximal size $r^{\prime} \sim R_{0}$.

We now close the parenthesis and return to the calculation of the cross-section in the BFKL approximation. When applied to the dipole-dipole cross-section $\sigma_{\mathrm{DD}}\left(r, r^{\prime}, \tau\right)$, the BFKL equation [四] reads (with $\bar{\alpha}_{s} \equiv \alpha_{s} N_{c} / \pi$ )

$$
\begin{aligned}
\frac{\partial}{\partial \tau} \sigma_{\mathrm{DD}}\left(r, r^{\prime}, \tau\right)=\frac{\bar{\alpha}_{s}}{2 \pi} \int & \mathrm{d}^{2} \boldsymbol{z} \frac{\boldsymbol{r}^{2}}{\boldsymbol{z}^{2}(\boldsymbol{r}-\boldsymbol{z})^{2}} \\
& \left\{-\sigma_{\mathrm{DD}}\left(r, r^{\prime}, \tau\right)+\sigma_{\mathrm{DD}}\left(z, r^{\prime}, \tau\right)+\sigma_{\mathrm{DD}}\left(|\boldsymbol{r}-\boldsymbol{z}|, r^{\prime}, \tau\right)\right\} .
\end{aligned}
$$

This equation has been written here as an evolution in the projectile size $r$ for a given target size $r^{\prime}$. (Of course, the function $\sigma_{\mathrm{DD}}\left(r, r^{\prime}, \tau\right)$ is symmetric in $r$ and $r^{\prime}$.) It has a simple physical interpretation: in one evolution step $(\tau \rightarrow \tau+\mathrm{d} \tau)$, the dipole $\boldsymbol{r}$ can split into two dipoles, with sizes $\boldsymbol{z}$ and respectively $\boldsymbol{r}-\boldsymbol{z}$, which then interact with the target. The 'BFKL kernel' $\frac{\bar{\alpha}_{s}}{2 \pi}\left[\boldsymbol{r}^{2} / \boldsymbol{z}^{2}(\boldsymbol{r}-\boldsymbol{z})^{2}\right]$ represents the differential probability for such a splitting to occur per unit rapidity. To solve Eq. (2.8), we also need an initial condition at low energy $(\tau=0)$. To the present accuracy, this is computed as the exchange of two gluons, which yields

$$
\sigma_{\mathrm{DD}}\left(r, r^{\prime}, \tau=0\right)=2 \pi \alpha_{s}^{2} r_{<}^{2}\left(1+\ln \frac{r_{>}}{r_{<}}\right)
$$

where $r_{<}=\min \left(r, r^{\prime}\right)$ and $r_{>}=\max \left(r, r^{\prime}\right)$. This cross-section shows 'color transparency' - it vanishes like $r_{<}^{2}$ when $r_{<} \rightarrow 0-$, meaning that a very small dipole scatters only weakly. By using Eq. (2.9), one can easily check that, for $\tau=0$ and $r \ll R_{0}$, the dipole-onium cross-section (2.5) is dominated by relatively large target dipoles, with $r \ll r^{\prime} \leq R_{0}$. (When $\Delta=0$, the large 
dipoles dominates over the small $\left(r^{\prime}<r\right)$ ones by a large logarithm $\ln \left(R_{0} / r\right)$.) As we shall later see, the feature is preserved by the evolution with increasing $\tau$.

The solution to the BFKL equation with the initial condition (2.9) is most conveniently obtained in Mellin space, where it reads

$$
\sigma_{\mathrm{DD}}\left(r, r^{\prime}, \tau\right)=\pi \alpha_{s}^{2} r^{2} \int_{C} \frac{\mathrm{d} \gamma}{2 \pi i} \frac{1}{\gamma^{2}(1-\gamma)^{2}}\left(\frac{r^{2}}{r^{\prime 2}}\right)^{1-\gamma} \mathrm{e}^{\tau \chi(\gamma)}
$$

where $\chi(\gamma)$ is the eigenvalue of the BFKL kernel in Mellin space, generally referred to as the $B F K L$ characteristic function

$$
\chi(\gamma)=\bar{\alpha}_{s}\{2 \psi(1)-\psi(\gamma)-\psi(1-\gamma)\}, \quad \psi(\gamma) \equiv \mathrm{d} \ln \Gamma(\gamma) / \mathrm{d} \gamma
$$

and the integration contour $C$ runs parallel to the imaginary axis with $0<\operatorname{Re}(\gamma)<1$. Note the symmetry property $\chi(\gamma)=\chi(1-\gamma)$, which reflects the conformal invariance of the BFKL evolution. In view of this, one can check that Eq. (2.10) is symmetric under $r \leftrightarrow r^{\prime}$, as it should.

The inverse Mellin transform in Eq. (2.10) can be evaluated via a saddle point approximation, in which the value of the saddle point depends upon the balance between the kinematical variables $\bar{\alpha}_{s} \tau$ and $\rho-\rho^{\prime}=\ln \left(r^{\prime 2} / r^{2}\right)$. Here, we have introduced the logarithmic variables $\rho \equiv \ln \left(R_{0}^{2} / r^{2}\right)$ and $\rho^{\prime} \equiv \ln \left(R_{0}^{2} / r^{\prime 2}\right)$ which are convenient for what follows. At this point, the reference scale inside the logarithms is arbitrary (it anyway cancels out in the difference $\rho-\rho^{\prime}$ ), but for later convenience we have chosen it as the onium size $R_{0}$.

Consider first the formal high-energy limit $\bar{\alpha}_{s} \tau \rightarrow \infty$ at fixed $\rho-\rho^{\prime}$ (this is formal since the respective solution violates the unitarity bound already for relatively low energies, as we shall shortly see). Then the saddle point $\gamma_{0}$ is determined by the minimum of the characteristic function :

$$
\chi^{\prime}\left(\gamma_{0}\right)=0 \quad \Longrightarrow \quad \gamma_{0}=1 / 2
$$

and Eq. (2.10) can be evaluated by expanding $\chi(\gamma)$ around $\gamma_{0}$, to second order ('diffusion approximation'): writing

$$
\gamma=1 / 2-i \nu
$$

one obtains (notice that $\chi(1 / 2-i \nu)$ is an even function of $\nu$, by conformal symmetry)

$$
\chi(1 / 2-i \nu)=\omega_{0}-D_{0} \nu^{2}+\mathcal{O}\left(\nu^{4}\right) \quad \text { with } \quad \omega_{0}=4 \ln 2 \bar{\alpha}_{s}, \quad D_{0}=14 \zeta(3) \bar{\alpha}_{s},
$$

and then the Gaussian integration over $\nu$ can be easily performed to yield

$$
\sigma_{\mathrm{DD}}\left(r, r^{\prime}, \tau\right) \simeq 8 \alpha_{s}^{2} \frac{\mathrm{e}^{\omega_{0} \tau}}{\sqrt{\pi D_{0} \tau}}\left(r^{2} r^{\prime 2}\right)^{1 / 2} \exp \left\{-\frac{\ln ^{2}\left(r^{2} / r^{\prime 2}\right)}{4 D_{0} \tau}\right\} .
$$

This expression exhibits the 'BFKL Pomeron', i.e., an exponential increase with $\tau$ at fixed $r$ and $r^{\prime}$. Clearly, when extrapolated at large $\tau$, this behaviour violates the unitarity bound. For instance, when $r \sim r^{\prime}$, this happens at a critical rapidity $\tau_{\text {cr }} \sim\left(1 / \omega_{0}\right) \ln \left(1 / \alpha_{s}^{2}\right)$, which is parametrically not that high. (Recall that the 'high-energy' regime starts only at $\tau \sim 1 / \bar{\alpha}_{s}$.) 
It is nevertheless possible to explore higher rapidities $\tau>\tau_{\text {cr }}$ while staying within the limits of the BFKL approximation provided we evolve along an oblique direction in the kinematical plane $\rho-\tau$ (see also Fig. 1) : when increasing $\tau$, we should simultaneously increase $\rho$ (i.e., decrease the ratio $\left.r / r^{\prime}\right)$, in such a way that the scattering amplitude remains small: $T\left(r, r^{\prime}, \tau\right) \ll$ 1. In particular, the saturation line $\rho=\rho_{s}(\tau)$ corresponds to a direction of evolution along which the amplitude is constant and of order one, cf. Eq. (2.7).

This also means that, in order to follow the saturation line, one must abandon the symmetry between the projectile and the target dipoles $\left(r \leftrightarrow r^{\prime}\right)$, which has been manifest so far. If the target dipole size is fixed to $r^{\prime}$, then with increasing $\tau$ we must evolve towards smaller and smaller values for $r$. It is then natural to write $\sigma_{\mathrm{DD}}\left(r, r^{\prime}, \tau\right)=2 \pi r^{\prime 2} T\left(r, r^{\prime}, \tau\right)$, where $r<r^{\prime}$ and the unitarity bound corresponds to $T\left(r, r^{\prime}, \tau\right)=1$. The BFKL approximation holds so long as $T\left(r, r^{\prime}, \tau\right) \ll 1$, but it allows us to approach the saturation line from small $r$ (large $\rho$ ) values, and thus compute the saturation momentum according to Eq. (2.7). To that aim, however, one cannot rely on the Pomeron saddle point, Eq. (2.12), anymore, rather one must determine the saddle point $\gamma_{s}$ corresponding to an evolution along the saturation line $\left(\rho=\rho_{s}(\tau)\right)$. By combining the saddle point condition:

$$
\tau \chi^{\prime}\left(\gamma_{s}\right)=-\left(\rho_{s}-\rho^{\prime}\right)
$$

with the condition that the amplitude 2.10 ) be approximately constant along the saturation line:

$$
\tau \chi\left(\gamma_{s}\right)-\left(1-\gamma_{s}\right)\left(\rho_{s}-\rho^{\prime}\right)-\ln \left(1 / \alpha_{s}^{2}\right)=0,
$$

(we have only kept the dominant parametric dependencies upon $\tau, \rho_{s}$ and $\alpha_{s}$ ), one obtains

$$
\begin{aligned}
\frac{\chi^{\prime}\left(\gamma_{s}\right)}{\chi\left(\gamma_{s}\right)} & =-\frac{1}{1-\gamma_{s}} \Longrightarrow \gamma_{s} \approx 0.372, \\
\rho_{s}\left(\tau, r^{\prime}\right) & =\rho^{\prime}+v_{s} \tau-\frac{\ln \left(1 / \alpha_{s}^{2}\right)}{1-\gamma_{s}} \quad \text { with } \quad v_{s} \equiv \frac{\chi\left(\gamma_{s}\right)}{1-\gamma_{s}} \approx 4.883 \bar{\alpha}_{s} .
\end{aligned}
$$

(We have assumed here that $\tau$, and hence $\rho_{s}$, are large enough for the term $\ln \left(1 / \alpha_{s}^{2}\right)$ to be treated as a small perturbation.) Note that $\gamma_{s}$ is a pure number, independent of either $\tau$ or $\bar{\alpha}_{s}$, corresponding to the fact that $\rho_{s}(\tau)$ is a straight line, with slope $v_{s} \sim \mathcal{O}\left(\bar{\alpha}_{s}\right)$.

For $\rho$ larger than $\rho_{s}$, but not much larger, one can estimate the amplitude by expanding Eq. (2.10) around $\gamma_{s}$ : writing $\gamma=\gamma_{s}-i \nu$, and expanding to second order in $\nu$, one finds

$$
\tau \chi(\gamma)-(1-\gamma) \rho-\ln \left(1 / \alpha_{s}^{2}\right) \simeq-\left(1-\gamma_{s}\right)\left(\rho-\rho_{s}\right)-i \nu\left(\rho-\rho_{s}\right)-D_{s} \tau \nu^{2},
$$

where $D_{s}=\chi^{\prime \prime}\left(\gamma_{s}\right) / 2 \approx 24.26 \bar{\alpha}_{s}$. This limited expansion is valid so long as $1<\rho-\rho_{s} \ll D_{s} \tau$. In this range, the amplitude is obtained by performing the Gaussian integration over $\nu$, as

$$
T\left(r, r^{\prime}, \tau\right) \simeq \frac{\left(r^{2} Q_{s}^{2}\right)^{1-\gamma_{s}}}{\sqrt{\pi D_{s} \tau}} \exp \left\{-\frac{\ln ^{2}\left(r^{2} Q_{s}^{2}\right)}{4 D_{s} \tau}\right\},
$$

where the saturation momentum (here, for a target dipole with size $r^{\prime}$ )

$$
Q_{s}^{2}\left(r^{\prime}, \tau\right)=\left(\alpha_{s}^{2}\right)^{1 /\left(1-\gamma_{s}\right)} \frac{1}{r^{\prime 2}} \mathrm{e}^{v_{s} \tau}
$$


appears as the natural reference scale for measuring the projectile dipole size. When viewed as a function of $r Q_{s}$, the amplitude near saturation, Eq. (2.20), is universal, i.e., independent of the specific properties of the target, but uniquely fixed by the evolution. Note that, in Eq. (2.20), $\gamma_{s}$ plays the role of an anomalous dimension. The evolution of the saturation momentum with increasing $\tau$, cf. Eq. (2.21), is universal as well, and controlled by the 'saturation exponent' $v_{s}$. The above calculation yields $v_{s} \sim \mathcal{O}(1)$ for $\bar{\alpha}_{s}=0.2 \div 0.3$, a rather large value that would be inconsistent with the phenomenology at HERA or RHIC. However, it turns out that, after taking into account the next-to-leading order BFKL corrections [2, 3], this value is reduced to $v_{s} \approx 0.3$ [31], which is indeed in the ballpark of the various phenomenological analyses (see, e.g., [34, 35, 36] for studies at HERA).

We now return to the problem of dipole-onium scattering, cf. Eqs. (2.5)-2.6), with the purpose of computing the corresponding saturation momentum $Q_{s}(\tau)$. As before, we shall approach the saturation line from the weak scattering regime, i.e., from relatively small dipole sizes $r \ll 1 / Q_{s}(\tau)$. This procedure meets with a subtle point though: although the overall scattering is, by assumption, weak, $T(r, \tau) \ll 1$, this is not necessarily so for all the individual dipoles which compose the onium and which contribute to the convolution in Eq. (2.5). The problem comes from the relatively small dipoles which, according to Eq. (2.21), develop large saturation momenta (meaning that they evolve into 'hot spots' with high gluon density), off which the projectile dipole can strongly scatter. Hence, when evaluating the contribution of such small dipoles to the total cross-section, one cannot rely on the BFKL approximation anymore. Still, as we shall shortly argue, this brings no serious complication, since, after being properly unitarized, the small dipoles give negligible contributions to the overall cross-section in the vicinity of the saturation line.

To see this, one can use the following, piecewise, approximation for the dipole-dipole crosssection at high energy,

$$
\sigma_{\mathrm{DD}}\left(r, r^{\prime}, \tau\right) \approx 2 \pi r_{>}^{2} \begin{cases}T\left(r_{<} Q_{s}\left(r_{>}, \tau\right), \tau\right) & \text { for } r_{<} \ll 1 / Q_{s}\left(r_{>}, \tau\right) \\ 1 & \text { for } r_{<} \gtrsim 1 / Q_{s}\left(r_{>}, \tau\right) .\end{cases}
$$

In the first line, $T\left(r_{<} Q_{s}\left(r_{>}, \tau\right), \tau\right)$ is the amplitude in the weak scattering regime, as given by Eqs. (2.20)-(2.21) after replacing $r \rightarrow r_{<}$and $r^{\prime} \rightarrow r_{>}$. The second line represents the black disk regime. The precise transition between these two regimes is not under control, but this is irrelevant for the present purposes. By inserting Eq. (2.22) into Eq. (2.5) and performing the integration over $r^{\prime}$, one can estimate the dipole-onium scattering amplitude $T(r, \tau) \equiv \sigma_{\text {dipole }}(r, \tau) / 2 \pi R_{0}^{2}$. The very small target dipoles with $r^{\prime}<r$ are easily seen to be irrelevant, since their contribution to $T(r, \tau)$ is of order $r^{2} / R_{0}^{2} \ll 1$. (Recall that we are only interested in contributions which can approach the unitarity limit $T=1$ when $r \rightarrow 1 / Q_{s}(\tau)$.) To evaluate the contribution of the larger dipoles with $r<r^{\prime} \leq R_{0}$, it is again convenient to use logarithmic variables $\left(\rho \equiv \ln \left(R_{0}^{2} / r^{2}\right)\right.$, etc.), and notice that Eq. (2.18) can be rewritten as

$$
\rho_{s}\left(\tau, r^{\prime}\right)=\rho^{\prime}+\rho_{s 0}(\tau), \quad \rho_{s 0}(\tau) \equiv v_{s} \tau-\frac{\ln \left(1 / \alpha_{s}^{2}\right)}{1-\gamma_{s}},
$$

where $\rho_{s 0}(\tau)$ represents the saturation momentum for a dipole with the maximal size $r^{\prime}=R_{0}$. We anticipate here that in the regime of interest we have $\rho>\rho_{s 0}(\tau)$. Then the contribution of 
the target dipoles with $\rho^{\prime}<\rho$ (i.e., $r^{\prime}>r$ ) can be evaluated as

$$
T(\rho, \tau) \simeq \int_{0}^{\rho-\rho_{s 0}} \mathrm{~d} \rho^{\prime} \mathrm{e}^{-\left(1-\gamma_{s}\right)\left(\rho-\rho^{\prime}-\rho_{s 0}\right)} \mathrm{e}^{-\frac{\left(\rho-\rho^{\prime}-\rho_{s 0}\right)^{2}}{4 D} D_{s}} \mathrm{e}^{-(1+\Delta) \rho^{\prime}}+\int_{\rho-\rho_{s 0}}^{\rho} \mathrm{d} \rho^{\prime} \mathrm{e}^{-(1+\Delta) \rho^{\prime}}
$$

where the first (second) term in the r.h.s. corresponds to large dipoles which scatters only weakly (respectively, to relatively small dipoles for which the unitarity limit has been reached). The first integral in the r.h.s. involves the overall exponential factor

$$
\mathrm{e}^{\left(1-\gamma_{s}\right) \rho^{\prime}} \mathrm{e}^{-(1+\Delta) \rho^{\prime}}=\mathrm{e}^{-\left(\Delta+\gamma_{s}\right) \rho^{\prime}}
$$

where the exponent $\Delta+\gamma_{s}$ is strictly positive in pQCD (recall that $\Delta \geq 0$ and $\gamma_{s} \approx 0.37$ ). It is then easy to check that, so long as $\rho-\rho_{s 0}<2\left(\Delta+\gamma_{s}\right) D_{s} \tau$, this integral is dominated by its lower limit $\rho^{\prime}=0$, i.e., by target dipoles of the largest possible size $r^{\prime} \sim R_{0}$. As for the second integral in Eq. (2.24), this is dominated by its own lower limit, at $\rho^{\prime}=\rho-\rho_{s 0}$. One thus finds

$$
T(\rho, \tau) \sim \frac{1}{\Delta+\gamma_{s}} \mathrm{e}^{-\left(1-\gamma_{s}\right)\left(\rho-\rho_{s 0}\right)} \mathrm{e}^{-\frac{\left(\rho-\rho_{s 0}\right)^{2}}{4 D_{s} \tau}}+\frac{1}{\Delta+1} \mathrm{e}^{-(1+\Delta)\left(\rho-\rho_{s 0}\right)} .
$$

As just mentioned, $\Delta+\gamma_{s}>0$, hence the second term in the r.h.s. is exponentially suppressed w.r.t. the first one. Thus, finally (up to a slowly varying prefactor),

$$
T(\rho, \tau) \sim \mathrm{e}^{-\left(1-\gamma_{s}\right)\left(\rho-\rho_{s 0}\right)} \mathrm{e}^{-\frac{\left(\rho-\rho_{s 0}\right)^{2}}{4 D_{s} \tau}} \quad \text { for } \quad \rho-\rho_{s 0}<2\left(\Delta+\gamma_{s}\right) D_{s} \tau,
$$

which is essentially the same result as for a target made with a single dipole of size $R_{0}$ (compare to Eq. (2.20)). In particular, the saturation momentum of the onium coincides with that of its largest dipole component (cf. Eq. (2.21)) $: \rho_{s}(\tau)=\rho_{s 0}(\tau)$, or

$$
Q_{s}^{2}(\tau)=\left(\alpha_{s}^{2}\right)^{1 /\left(1-\gamma_{s}\right)} \frac{1}{R_{0}^{2}} \mathrm{e}^{v_{s} \tau}
$$

This is quite intuitive: the onium starts to look 'black' as a whole only when a large dipole, which covers all (or most) of the hadron disk, has evolved into a system with high gluon density on the resolution scale of the projectile. Smaller dipoles reach the unitarity limit much faster (on that particular resolution scale), but their contributions to the total cross-section are suppressed by their small area ${ }^{1}$.

The high-energy dipole amplitude in Eqs. (2.20) or (2.27) exhibits an interesting structure, which can be better appreciated by comparison with the low-energy amplitude (2.9), or the (purely) BFKL prediction at high energy, Eq. (2.15): Besides the power-law $r^{2\left(1-\gamma_{s}\right)}$ with anomalous dimension $\gamma_{s} \approx 0.37$, there is also a Gaussian factor describing diffusion in the logarithmic variable $\rho \sim \ln \left(1 / r^{2}\right)$. This shows that, when increasing $\tau$, the amplitude gets built via a diffusive process which gets most of its support from the region $0<\rho-\rho_{s}<\sqrt{4 D_{s} \tau}$ above the saturation line. For $\tau$ large enough, such that $Q_{s}^{2}(\tau) \gg \Lambda_{\mathrm{QCD}}^{2}$, this region lies fully in the perturbative domain, thus confirming the internal consistency of our present approach. The physics of saturation/unitarization eliminates the symmetric diffusion characteristic of the (pure) BFKL evolution, cf. Eq. (2.15), which would invalidate the use of perturbation theory.

\footnotetext{
${ }^{1}$ Of course, the physical picture would be different if $\Delta$ was negative and large enough, in such a way to bias the dipole distribution (2.6) towards small sizes. Then, the onium could become black as a whole (on a given resolution scale) by getting covered with many small dipoles which are individually black.
} 


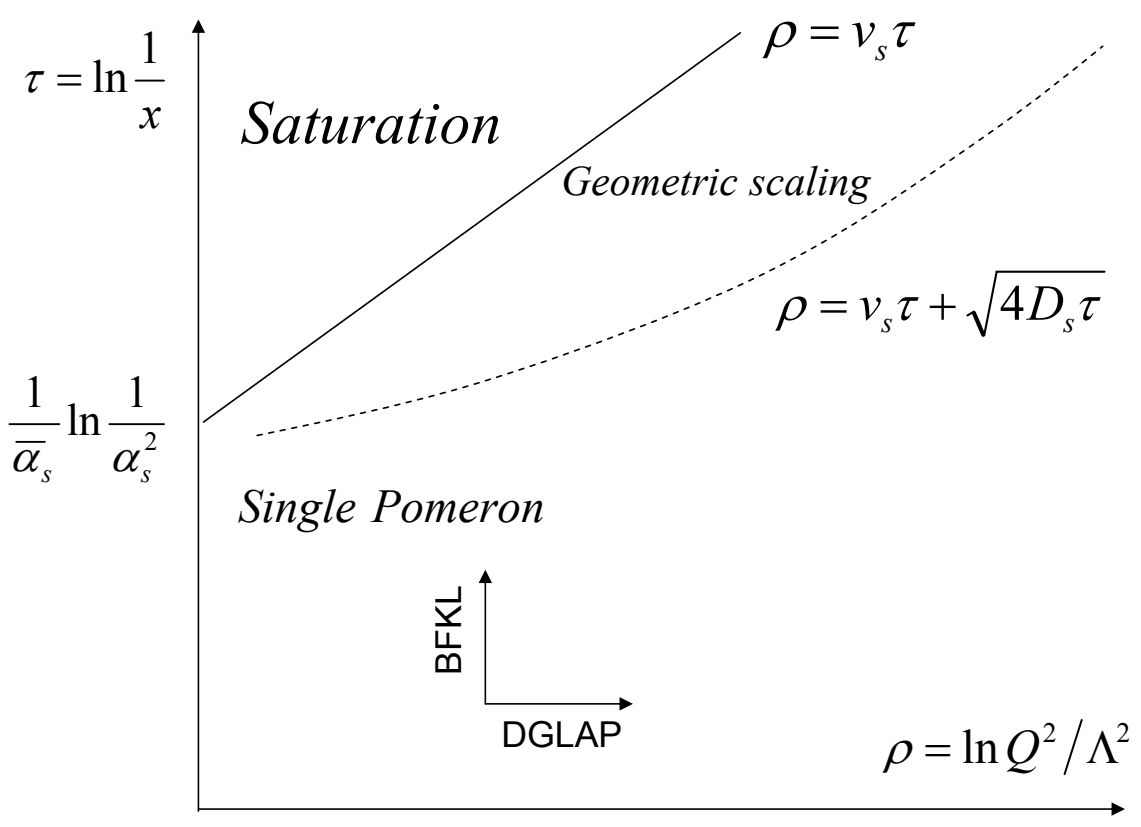

Figure 1: A 'phase diagram' for the high energy evolution in $p Q C D$ in the presence of unitarity corrections and gluon saturation. (We recall that $\bar{\alpha}_{s} \equiv \alpha_{s} N_{c} / \pi$.)

In particular, within the more restricted window $\rho-\rho_{s} \ll \sqrt{4 D_{s} \tau}$ (close to the saturation line), the diffusion term in Eq. (2.27) can be ignored, and then the amplitude shows geometric scaling, i.e., it depends upon $r$ and $\tau$ only via the single variable $r Q_{s}(\tau)$ :

$$
T(r, \tau) \sim \mathrm{e}^{-\left(1-\gamma_{s}\right)\left(\rho-\rho_{s}\right)}=\left(r^{2} Q_{s}^{2}(\tau)\right)^{1-\gamma_{s}} \quad \text { for } \quad \rho-\rho_{s} \ll \sqrt{4 D_{s} \tau} .
$$

This scaling property is an important consequence of the non-linear dynamics responsible for unitarization on the shape of the amplitude in the weak scattering regime 'above saturation' $\left(\rho>\rho_{s}\right)$. Via the dipole factorization $(2.3)$, this property gets transmitted to the DIS crosssection in the high $Q^{2}$ regime at $Q^{2}>Q_{s}^{2}(\tau)$ :

$$
\frac{F_{2}\left(Q^{2}, \tau\right)}{Q^{2}} \sim \sigma_{\gamma^{*} p}\left(Q^{2}, \tau\right) \sim N_{c} R_{0}^{2}\left(\frac{Q_{s}^{2}(\tau)}{Q^{2}}\right)^{1-\gamma_{s}} \quad \text { for } \quad Q_{s}^{2}(\tau) \lesssim Q^{2} \ll Q_{g}^{2}(\tau),
$$

where $Q_{g}^{2}(\tau) \equiv Q_{s}^{2}(\tau) \mathrm{e}^{\sqrt{4 D_{s} \tau}}$ is the upper bound of the scaling window, cf. Eq. 2.29$)$.

It is furthermore interesting to note the behaviour of $F_{2}$ for very small values of $Q^{2}$, deeply at saturation $\left(Q^{2} \ll Q_{s}^{2}(\tau)\right)$. Then, the virtual photon cross-section is dominated by large dipoles, with $1 / Q_{s}^{2}(\tau)<r^{2}<1 / Q^{2}$, for which the black disk limit is saturated: $\sigma_{\text {dipole }}(r, \tau) \approx 2 \pi R_{0}^{2}$. One then finds (the logarithm is generated by the integral over $r^{2}$ )

$$
\frac{F_{2}\left(Q^{2}, \tau\right)}{Q^{2}} \sim N_{c} R_{0}^{2} \ln \frac{Q_{s}^{2}(\tau)}{Q^{2}} \quad \text { for } \quad Q^{2} \ll Q_{s}^{2}(\tau),
$$

which exhibits geometric scaling too. Remarkably, it turns out that a similar scaling has been observed indeed [35] in the HERA data for DIS at small $x \leq 0.01$ and up to relatively large 
values of $Q^{2} \sim 100 \mathrm{GeV}^{2}$ (well above the saturation momentum at HERA, estimated in the ballpark of $1 \mathrm{GeV}$ ). The 'phase-diagram' for high energy evolution in pQCD which emerges from the previous considerations is graphically summarized in Fig. 1.

\section{DIS at strong coupling}

\subsection{The setup}

With this section we begin the study of the most interesting problem for us here, namely DIS at strong 't Hooft coupling and high energy. Specifically, our physical gauge theory will be a deformation of the $\mathcal{N}=4 \mathrm{SYM}$ theory in the regime where the gauge coupling is weak, $g \ll 1$, but the number of colors is large, $N_{c} \gg 1$, in such a way that the 't Hooft coupling $\lambda \equiv g^{2} N_{c}$ (the relevant coupling for perturbation theory at large $N_{c}$ ) is large : $\lambda \gg 1$. The 'deformation' refers to the introduction of an effective infrared cutoff which breaks down conformal symmetry and mimics confinement.

According to the AdS/CFT correspondence [11], the $\mathcal{N}=4$ SYM theory is admitted to have a dual description in terms of the Type IIB string theory living in the 10-dimensional $A d S_{5} \times S^{5}$ space-time, with the following metric:

$$
\mathrm{d} s^{2}=\frac{u^{2}}{R^{2}}\left(-\mathrm{d} x_{0}^{2}+\mathrm{d} x_{1}^{2}+\mathrm{d} x_{2}^{2}+\mathrm{d} x_{3}^{2}\right)+\frac{R^{2}}{u^{2}} \mathrm{~d} u^{2}+R^{2} \mathrm{~d} \Omega_{5}^{2} .
$$

In this equation, $x_{0}$ and $\boldsymbol{x}=\left(x_{1}, x_{2}, x_{3}\right)$ are the time and, respectively, three spatial dimensions of 'our' physical Minkowski world, $u$ is the $A d S_{5}$ radial coordinate $(0 \leq u<\infty)$, to be below referred as 'the 5th dimension', and $\mathrm{d} \Omega_{5}^{2}$ is the angular measure on $S^{5}$. Furthermore, $R$ is the common radius of $A d S_{5}$ and $S^{5}$, and it is related to the string length $l_{s}$ via the 't Hooft coupling of the dual gauge field theory: $R=l_{s} \lambda^{\frac{1}{4}}=\alpha^{\prime \frac{1}{2}} \lambda^{\frac{1}{4}}$, with $\alpha^{\prime} \equiv l_{s}^{2}$ the Regge slope. The physical, 4-dimensional, space is identified with the boundary of $A d S_{5}$ at $u \rightarrow \infty$, where the $A d S_{5}$-metric becomes conformal to the standard Minkowski metric $\eta^{\mu \nu}$. The string coupling constant $g_{s}$ and the gauge coupling $g$ are related to each other as $g_{s}=g^{2} / 4 \pi=\lambda / 4 \pi N_{c}$, which shows that in the 'strong coupling' regime of interest here $(\lambda \gg 1$ but $g \ll 1)$, the string theory is weakly coupled and hence it can be treated in a semi-classical approximation.

We have previously mentioned the 'deformation' of the $\mathcal{N}=4$ SYM theory which is necessary to mimic confinement. In the dual string theory, this translates into a modification of the metric at small $u \sim u_{0} \equiv \Lambda R^{2}$, where $\Lambda$ is a parameter which sets the scale of light hadrons ('glueballs'). The precise way of modifying the geometry in the infrared is an issue of debate which we do not enter. (As we shall see, the dominant behaviour at high energy is insensitive to such details.) In what follows, we shall simply restrict the 5 th coordinate to values $u \geq u_{0}$ (in practice, we shall be mostly interested in $u \gg u_{0}$ ), and assume that the spectrum of the supergravity theory includes states which are localized near $u=u_{0}$, corresponding to massive 'glueballs' on the gauge theory side. Note that, because of the logarithmic nature of the metric in $u\left(\mathrm{~d} s_{u}^{2} \propto \mathrm{d} u^{2} / u^{2}\right)$, massive 'supergravity' states exhibit power-law decays at large $u$, to be better characterized later on.

To closely mimic the setup of DIS, we follow [15] and introduce a 'photon' as the gauge boson associated with a gauged $U(1)$ subgroup of the $S U(4) \mathcal{R}$-symmetry. As for the target, we employ a 'supergravity' glueball of mass $\sim \Lambda$ which originates from the Kaluza-Klein decomposition 
of the 10 dimensional massless dilaton after compactification on $S^{5}$. Still as in Ref. [15], we shall use indices $M, N, \ldots$ to denote all ten space-time dimensions, separating into $\mu, \nu, \ldots$ on the Minkowski boundary at $u \rightarrow \infty$ (our physical space), $m, n, \ldots$ on $A d S_{5}$, and $a, b, \ldots$ on $S^{5}$. Indices are raised or lowered in the standard way, using the metric $G_{M N}$ which can be read off Eq. (3.1) : $\mathrm{d} s^{2}=G_{M N} \mathrm{~d} x^{M} \mathrm{~d} x^{N}$. It is understood that for quantities living in the fourdimensional gauge theory (and hence also on the Minkowski boundary of $A d S_{5}$ ), the tensor operations are performed with the Minkowski metric $\eta^{\mu \nu}=(-1,1,1,1)$.

As standard in DIS, the structure functions are obtained from the imaginary part of the forward Compton scattering amplitude

$$
i \int \mathrm{d}^{4} x \mathrm{e}^{i q \cdot x}\left\langle P\left|T\left\{J^{\mu}(x) J^{\nu}(0)\right\}\right| P\right\rangle,
$$

where $J^{\mu}(x)$ is the density of the $\mathcal{R}$-current and $|P\rangle$ denotes a (normalizable) glueball state with 4-momentum $P^{\mu}$. The hadronic matrix element under the integral will be computed from semi-classical string theory, via the AdS/CFT correspondence. The recipe is as follows :

The $\mathcal{R}$-current at the Minkowski boundary $(u \rightarrow \infty)$ excites vector-like metric fluctuations in the 'bulk' (i.e., at finite $u \geq u_{0}$ ) :

$$
\delta G_{m a}\left(x_{\mu}, u, \Omega\right)=A_{m}\left(x_{\mu}, u\right) v_{a}(\Omega),
$$

where $\Omega$ denotes the angular coordinates on $S^{5}, A_{m}\left(x_{\mu}, u\right)$ is a gauge boson field, and $v_{a}$ is the Killing vector on $S^{5}$ corresponding to the gauged $U(1)$ subgroup. At $u \rightarrow \infty$, the field $A_{\mu}\left(x_{\mu}, \infty\right)$ can be identified as the physical photon which couples to the $\mathcal{R}$-current. This will be taken in the form $A_{\mu}\left(x_{\mu}, \infty\right)=n_{\mu} \mathrm{e}^{i q \cdot x}$, with space-like four-vector $q^{\mu}: Q^{2} \equiv q^{\mu} q_{\mu}>0$. But inside the bulk, $A_{m}\left(x_{\mu}, u\right)$ should be better viewed as a gravitational wave, as it describes a fluctuation in the metric tensor. For definiteness, we shall refer to this field as the ' $\mathcal{R}$-boson'. This $\mathcal{R}$-boson propagates into the bulk until it scatters off the dilaton target - a supergravity state with wavefunction

$$
\Psi\left(x_{\mu}, u, \Omega\right)=\mathrm{e}^{i P \cdot x} \Phi(u, \Omega)
$$

Note that this is a plane wave with four-momentum $P^{\mu}$ in the four physical dimensions. The Bjorken- $x$ variable for DIS is defined in the usual way: $x \equiv Q^{2} /(2 P \cdot q)$. Still as usual, the high-energy limit $\left(P \rightarrow \infty\right.$ at fixed $\left.Q^{2}\right)$ corresponds to small values for $x(x \ll 1)$.

As explained in Refs. [15], the Regge behavior starts to be seen when $1 / x \gg \sqrt{\lambda}$ where the energy is high enough to create excited string intermediate states. To leading order at large $N_{c}$, the string scattering can be computed at tree level, as the imaginary part of the superstring Virasoro-Shapiro amplitude for graviton-dilaton scattering [23] (originally calculated in flat space, and heuristically extended to curved space in [15]) convoluted with the supergravity wavefunctions for the $\mathcal{R}$-boson $\left(A_{m}\right)$ and the dilaton $(\Phi)$. The result of [15] can be compactly written as

$$
F_{2}\left(x, Q^{2}\right)=c \alpha^{\prime} \frac{(Q R)^{6}}{\Lambda^{2}} \int \frac{\mathrm{d} u}{u} \frac{1}{u^{4}}\left(K_{0}^{2}\left(Q R^{2} / u\right)+K_{1}^{2}\left(Q R^{2} / u\right)\right)\left(\frac{1}{x}\right)^{1+\frac{\alpha^{\prime} \Delta_{2}}{2}} \Phi^{\dagger} \Phi(u),
$$


where we have absorbed in $c$ a constant coming from the integration over $S^{5}$, as well as powers of $\lambda$. (For simplicity, we do not keep track of powers of $\lambda$ in the prefactor. This is acceptable since we shall deal with an exponentially large factor $\mathrm{e}^{\sqrt{\lambda}}$. See below for details.)

Let us discuss the various factors under the integral in Eq. (3.5) one after the other:

(i) The modified Bessel functions $K_{0,1}$ are a part of the ' $\mathcal{R}$-boson wavefunction'; that is, they arise via the solution $A_{m}\left(x_{\mu}, u\right)$ to the supergravity equation of motion for the metric perturbation induced by the $\mathcal{R}$-current. This equation is Maxwell equation on $\operatorname{Ad}_{5}: D^{m} F_{m n}=0$, with $F_{m n}=\partial_{m} A_{n}-\partial_{n} A_{m}$ and $D^{m}$ the covariant derivative in the $A d S_{5}$ background. $\left(A_{m}\left(x_{\mu}, u\right)\right.$ is the non-normalizable solution to this equation corresponding to the boundary condition $A_{\mu}\left(x_{\mu}, u \rightarrow \infty\right)=n_{\mu} \mathrm{e}^{i q \cdot x}$.) For $u$ smaller than

$$
u_{c} \equiv Q R^{2}
$$

the Bessel functions decays exponentially, meaning that a 'photon' with large $Q^{2}$ cannot penetrate deeply inside the bulk: the larger $Q^{2}$ is, the closer the photon remains near the boundary. Since, on the other hand, the inverse power of $u$ manifest in Eq. (3.5) favors small values of $u$, it is clear that the integral there is controlled by $u \sim u_{c}$.

Remarkably, the $\mathcal{R}$-boson wavefunction involves the same Bessel functions as the wavefunction describing the $q \bar{q}$ excitation of a virtual photon in pQCD (cf. Eq. (2.4)). This suggests a kind of 'duality' between the dipole size $r$ in pQCD and the 5th dimension $u$ in $A d S_{5}$, via the correspondence $r \leftrightarrow R^{2} / u$. We shall identify further aspects of this correspondence later on.

(ii) The dilaton wavefunction $\Phi(u)$ is peaked around $u \sim u_{0}$ and decays at large $u$ like $\Phi \sim u^{-\Delta}$, where $\Delta$ is the conformal dimension of the operator which creates this state. For glueballs corresponding to supergravity modes, $\Delta$ is a number of $\mathcal{O}(1)$ which is strictly larger than 2. In the formal analogy to pQCD, the probability density $|\Phi(u)|^{2} \sim u^{-2 \Delta}$ corresponds to the density $n(r)$ of dipoles inside the onium, cf. Eq. (2.6). Note that the overlap between the $\mathcal{R}$-boson and the dilaton wavefunction scales like $u_{c}^{-2 \Delta} \sim\left(1 / Q^{2}\right)^{\Delta}$, and hence it rapidly dies away when increasing $Q^{2}$.

(iii) The last comment shows that DIS would be strongly suppressed at large $Q^{2}$ if there was not for the non-locality hidden in the middle factor, $(1 / x)^{1+\left(\alpha^{\prime} / 2\right) \Delta_{2}}$, which describes the imaginary part associated with one string exchange in the curved, $A d S_{5}$, space. In less formal notations, the action of this operator can be written as (with $\tau \equiv \ln (1 / x)$ )

$$
\left(\frac{1}{x}\right)^{1+\frac{\alpha^{\prime} \Delta_{2}}{2}} \Phi^{\dagger} \Phi(u) \equiv \frac{1}{x} \int \mathrm{d} u^{\prime}\left\langle u\left|\mathrm{e}^{\frac{\alpha^{\prime} \tau \Delta_{2}}{2}}\right| u^{\prime}\right\rangle\left|\Phi\left(u^{\prime}\right)\right|^{2} .
$$

Notice the dominant energy behavior $F_{2}(x) \sim 1 / x$ at small $x$ : this is the hallmark of the graviton exchange in the $t$-channel. (At high energy, the exchanged string is dominated by its graviton component; see Sect. 4.2.) Furthermore, the operator $\Delta_{2}$ in the exponent is the Laplacian describing diffusion in the 5 th dimension, for the spin-2 exchanged graviton. This operator appears because of the finite momentum transfer in the $u$-direction, although the momentum transfer in the physical four dimensions is strictly zero [15, 16]. Explicitly, this operator reads

$$
\Delta_{2} \equiv\left(\frac{u}{R}\right)^{2} \nabla_{0}^{2}\left(\frac{u}{R}\right)^{-2}=\frac{4}{R^{2}}\left(\partial_{\rho}^{2}-1\right)
$$


where $\nabla_{0}^{2}$ is the scalar Laplacian on $A d S_{5}$ and we have defined $\rho \equiv \ln \left(u^{2} / u_{0}^{2}\right)$. By also using the integral representation of the 'heat kernel' $\exp \left\{\partial_{\rho}^{2}\right\}$, one arrives at the following representation for the expression in Eq. (3.7) :

$$
\begin{aligned}
\mathrm{e}^{\tau+2 \alpha^{\prime} \tau\left(\partial_{\rho}^{2}-1\right)} \Phi^{\dagger} \Phi(\rho) & =\int \mathrm{d} \rho^{\prime} \int \frac{\mathrm{d} \nu}{2 \pi} \mathrm{e}^{i \nu\left(\rho-\rho^{\prime}\right)} \mathrm{e}^{\tau-\frac{2 \tau}{\sqrt{\lambda}}\left(\nu^{2}+1\right)}\left|\Phi\left(\rho^{\prime}\right)\right|^{2} \\
& =\frac{\mathrm{e}^{\omega_{0} \tau}}{2 \sqrt{\pi D \tau}} \int_{0}^{\infty} \mathrm{d} \rho^{\prime} \mathrm{e}^{\frac{-\left(\rho-\rho^{\prime}\right)^{2}}{4 D \tau}} \mathrm{e}^{-\Delta \rho^{\prime}},
\end{aligned}
$$

where we have used $R^{2} / \alpha^{\prime}=\sqrt{\lambda}$ and introduced

$$
\omega_{0} \equiv 1-\frac{2}{\sqrt{\lambda}}, \quad \text { and } \quad D \equiv \frac{2}{\sqrt{\lambda}} .
$$

Eq. (3.9) result shows a Pomeron-like increase with $\tau$, with an intercept $1+\omega_{0}=2-2 / \sqrt{\lambda}$ which is slightly shifted from 2 due to the curvature of $A d S_{5}$ [14, 15, 16].

One should notice the formal similarity between the stringy 'Pomeron' amplitude in Eq. (3.9) and the cross-section (2.15) which describes the BFKL Pomeron in pQCD. In this analogy, the fifth coordinate $u$ plays, once again, the role of the inverse dipole size $1 / r$. Like for the BFKL Pomeron, the non-locality associated with the single Pomeron exchange in $A d S_{5}$ is diffusive in $\rho \sim \ln u^{2}$, with the rapidity $\tau$ playing the role of the evolution 'time'; accordingly, its effect becomes important only for very high energies, exponentially large in the relevant coupling. Namely, the diffusive radius $D \tau$ in Eq. (3.9) becomes of $\mathcal{O}(1)$ when $\tau \sim \sqrt{\lambda}$, or $1 / x \sim \mathrm{e}^{\sqrt{\lambda}}$. For comparison, the corresponding estimate in pQCD reads $\tau \sim 1 / \bar{\alpha}_{s}$, or $1 / x \sim \mathrm{e}^{1 / \bar{\alpha}_{s}}$, which at a first sight may look as an astronomically high energy, but in fact is is not: $\bar{\alpha}_{s}$ is not that small in real world $\left(\bar{\alpha}_{s}=0.2 \div 0.4\right.$ for the high-energy experiments), and rapidities of order $1 / \bar{\alpha}_{s}$ or even larger are within the reach of the present-day accelerators.

Up to an overall factor of $\mathcal{O}\left(1 / N_{c}^{2}\right)$ that we shall shortly comment on, Eq. (3.9) can be viewed as the string theory analog of the dipole-onium scattering amplitude in $\mathrm{pQCD}$, in the BFKL approximation. The dipole (a $q \bar{q}$ excitation of the virtual photon with a given transverse size $r$ ) is now replaced by the $\mathcal{R}$-boson $A_{m}(u)$ (the metric fluctuation induced by the $\mathcal{R}$-current at a given position $u$ along the 5 th dimension), and the dilaton plays the same role as the onium - the corresponding wavefunctions are localized near the respective 'infrared' cutoff $\left(u \sim u_{0}\right.$ for the dilaton and, respectively, $r \sim R_{0}$ for the onium) and they exhibit power-law tails with exponent $\Delta$ in the 'ultraviolet' (large $u$ and, respectively, small $r$ ). At this stage, it would be straightforward to repeat the analysis in Sect. 2 (mutatis mutandis) and thus determine the saturation momentum for the dilaton. However, before doing that, it is necessary to better understand the validity limits of the approximation in Eq. (3.9). We shall do that in Sect. 3.2, where we shall discover that, in the string theory context, Eq. (3.9) is less general than its analog in pQCD - it holds only within a limited range of values for $\tau$ and $\rho$. These limitations have not been properly recognized in the previous literature.

We conclude this subsection with a discussion of the interplay between the large $-N_{c}$ limit and the high-energy limit, which turns out to be quite subtle, for both weak and strong coupling. The tree-level string amplitude (corresponding to single Pomeron exchange) is of order $g_{s}^{2} \propto 1 / N_{c}^{2}$. In Eq. (3.5) this factor $1 / N_{c}^{2}$ has been canceled by a factor $N_{c}^{2}$ implicit in the $\mathcal{R}$-current. (The 
latter receives contributions from all the fields in the $\mathcal{N}=4$ SYM theory which are charged under the $\mathcal{R}$-symmetry; these fields are scalars and Weyl fermions in the adjoint representation of the colour group, hence they have $N_{c}^{2}-1 \approx N_{c}^{2}$ degrees of freedom.)

This is similar to what happens in QCD at weak coupling: the BFKL result (2.10) involves an overall factor $\alpha_{s}^{2} \sim \bar{\alpha}_{s}^{2} / N_{c}^{2}$ which is partially canceled in $F_{2}$ by a factor $N_{c}$ coming from the photon wavefunction, Eq. (2.4). Yet, the unitarity corrections apply directly to the dipoledipole scattering (and not only to $F_{2}$ ), hence they become important when the small factor $1 / N_{c}^{2}$ manifest in Eqs. (2.10) or (2.15) gets compensated by the energy evolution. As discussed below Eq. (2.15), for the BFKL Pomeron this happens at a critical rapidity $\tau_{\mathrm{cr}} \sim\left(1 / \bar{\alpha}_{s}\right) \ln \left(N_{c}^{2} / \bar{\alpha}_{s}^{2}\right)$. For $\tau \gtrsim \tau_{\mathrm{cr}}$, multiple Pomeron exchanges become important and ensure unitarization.

Returning to strong coupling and the dual string theory, there the multiple Pomeron exchanges correspond to higher genus string amplitudes which are suppressed by powers of $1 / N_{c}^{2}$. Hence, unitarity corrections become important at $\tau_{\mathrm{cr}} \sim \ln N_{c}^{2}$. We thus see that, in the strict large $-N_{c}$ limit $\left(N_{c} \rightarrow \infty\right.$ at fixed energy), there is no issue of unitarity: higher loop amplitudes, being proportional to inverse powers of $1 / N_{c}^{2}$, can be made arbitrarily small even at very high energy. On the other hand, when $N_{c}$ is large but finite, the single Pomeron exchange has only a limited applicability, and beyond that one has to sum over an infinite number of multi-loop diagrams corresponding to arbitrarily many Pomerons exchanged in the $t$-channel. What is however different from the situation at weak coupling is that, for generic values of $\tau$ and $\rho \sim \ln Q^{2}$, the amplitude in the vicinity of the unitarity line is not dominated by the single Pomeron exchange anymore. This is why, in general, the saturation line cannot be inferred from the previous formulæ in this section, but requires some further analysis.

It will be our goal in what follows to determine the onset of the various types of unitarity corrections in the parameter space of $\tau$ and $\rho$. To have access to the full structure of the highenergy 'phase diagram', we shall assume that $N_{c}^{2}>\mathrm{e}^{\sqrt{\lambda}}-$ so that diffusion effects (which, we recall, require a rapidity evolution $\tau \gtrsim \sqrt{\lambda}$ ) become important before unitarization. But the opposite case $\mathrm{e}^{\sqrt{\lambda}} \ll N_{c}^{2}$ will be included too in our results, as a special limit.

\subsection{Limit of the single Pomeron approximation : A lesson from a sum rule}

The fundamental difference between the physics of unitarization at weak and, respectively, strong coupling comes from the fact that, in the strongly-coupled gauge theories with a gravity dual, the high-energy scattering amplitude is predominantly real (it starts as one graviton exchange), while at weak coupling it is predominantly imaginary (in both QCD and $\mathcal{N}=4$ SYM theory). Of course, the large real part at strong coupling is irrelevant for the total cross section so long as the latter is computed at tree-level. But at higher loop level, say, starting with two-graviton exchange, this gives rise to an imaginary part via diffractive processes - i.e., processes where the final states (string excitations or higher Kaluza-Klein modes) are separated by a rapidity gap. On the other hand, the imaginary part of the tree-level amplitude (3.5) is associated with the inelastic production of excited string states, with no rapidity gap ${ }^{2}$. It turns out that, when $\rho \sim \ln Q^{2}$ is larger than a certain critical value, the diffractive processes dominate over

\footnotetext{
${ }^{2}$ Strictly speaking, the DIS process is always inelastic, since the virtual photon cannot appear in the final state. Here, we use the word 'inelastic' to suggestively distinguish the final states without rapidity gap from the diffractive ones, which exhibit such a gap.
} 
the inelastic ones in the approach towards unitarity. We shall discuss this issue in detail in the next section, but for the time being let us proceed naively and study the onset of unitarity corrections on the basis of the tree-level results in the previous subsection, which include the inelastic processes alone. This will drive us into a paradox which will point out towards the necessity to include the additional, diffractive, contributions.

After also including the essential factor $1 / N_{c}^{2}$, the forward scattering amplitude (3.9) for the $\mathcal{R}$-boson-dilaton can be more suggestively rewritten as (compare to Eq. (2.5))

$$
T(\rho, \tau)=\int_{0}^{\infty} \mathrm{d} \rho^{\prime} T_{0}\left(\rho, \rho^{\prime}, \tau\right)\left|\Phi\left(\rho^{\prime}\right)\right|^{2}
$$

where $\left|\Phi\left(\rho^{\prime}\right)\right|^{2}=\mathrm{e}^{-\Delta \rho^{\prime}}$ and

$$
T_{0}\left(\rho, \rho^{\prime}, \tau\right) \equiv \frac{1}{N_{c}^{2}} \frac{\mathrm{e}^{\omega_{0} \tau}}{2 \sqrt{\pi D \tau}} \mathrm{e}^{-\frac{\left(\rho-\rho^{\prime}\right)^{2}}{4 D \tau}}
$$

represents the elementary scattering amplitude between states localized around $\rho$ and $\rho^{\prime}$ in the single-Pomeron-exchange approximation (the analog of the dipole-dipole amplitude in Sect. 2). The unitarity bound on this elementary amplitude determines the saturation line for a dilaton state localized at $\rho^{\prime}$; namely, the condition $T_{0}\left(\rho, \rho^{\prime}, \tau\right) \sim 1$ for $\rho=\rho_{s}\left(\tau, \rho^{\prime}\right)$ implies

$$
\rho_{s}\left(\tau, \rho^{\prime}\right)=\rho^{\prime}+\rho_{s 0}(\tau), \quad \rho_{s 0}(\tau) \equiv \sqrt{4 D \tau\left(\omega_{0} \tau-\ln N_{c}^{2}\right)},
$$

where $\rho_{s 0}(\tau)$ refers to a dilaton state localized at $u \sim u_{0}$. (As in Sect. 2 , we neglect the effects of the slowly varying prefactor $1 /(2 \sqrt{\pi D \tau})$ in Eq. (3.12).)

Eq. (3.13) applies for $\tau>\tau_{\mathrm{cr}}=\left(1 / \omega_{0}\right) \ln N_{c}^{2}$ and should be compared to the corresponding result, Eq. (2.23), in pQCD: the similarity between these two results would become even closer if it was possible to extrapolate Eq. (3.13) to large rapidities $\tau \gg \tau_{\mathrm{cr}}$, where $\ln N_{c}^{2}$ could be expanded out from the square root. However, as we shall later discover, such an extrapolation would be incorrect: in reality, Eq. (3.13) applies only within a limited range in $\tau$ above $\tau_{\text {cr }}$, namely, within the window $\tau_{\text {cr }}<\tau<\tau_{c}$, with $\tau_{c}-\tau_{\text {cr }} \simeq 2 \ln N_{c}^{2} / \sqrt{\lambda}$ (see Eq. (3.28) below).

This limitation is in turn related to a failure of our formula (3.12) for the elementary amplitude, which becomes incomplete at high energies and for large values of $\rho$. To illustrate this failure, we shall show that Eq. (3.12) leads to an unphysical result when used to evaluate the following moment of the structure function

$$
M\left(Q^{2}\right) \equiv \int_{0}^{1} \mathrm{~d} x F_{2}\left(x, Q^{2}\right)=\int_{0}^{\infty} \mathrm{d} \tau \mathrm{e}^{-\tau} F_{2}\left(\tau, Q^{2}\right) .
$$

By energy-momentum conservation, this moment should be independent of $Q^{2}$; however, as we shall demonstrate in what follows, the (properly unitarized) amplitude for single Pomeron exchange, Eq. (3.12), fails to saturate the sum-rule at large values of $Q^{2}$.

Within the present approximations, the structure function $F_{2}$ of the dilaton is given by Eq. (3.5). To avoid unnecessary complications with the $\mathcal{R}$-boson wavefunction, we shall here restrict ourselves to the structure function that would be measured by a single $\mathcal{R}$-boson state localized at $u$ (the analog of a single dipole projectile with fixed size $r$ in pQCD). The coordinate $u$ fixes the resolution scale, $u^{2} \propto Q^{2}$ (cf. Eq. (3.6)), hence $F_{2}\left(\tau, Q^{2}\right)$ can be evaluated as

$$
F_{2}\left(\tau, Q^{2}\right) \sim N_{c}^{2} \mathrm{e}^{\rho} T(\rho, \tau)
$$


(up to an irrelevant prefactor), with $\rho \equiv \ln \left(u^{2} / u_{0}^{2}\right)$. The factor of $N_{c}^{2}$ accounts for the color degrees of freedom of the fields contributing to the $\mathcal{R}$-current and $\mathrm{e}^{\rho}$ accounts for the factor $Q^{2}$ in the relation (2.2) between $F_{2}$ and the 'dipole' (here, $\mathcal{R}$-boson) cross-section.

We start by evaluating $T(\rho, \tau)$ in the single-Pomeron approximation. This is given by Eq. (3.11) with the elementary amplitude from Eq. (3.12) corrected for unitarity violations. That is, for $\tau>\tau_{\mathrm{cr}}$, we shall write (compare to Eq. (2.24) in pQCD)

$$
T(\rho, \tau) \simeq \int_{0}^{\rho-\rho_{s 0}} \mathrm{~d} \rho^{\prime} \frac{1}{N_{c}^{2}} \mathrm{e}^{\omega_{0} \tau} \mathrm{e}^{-\frac{\left(\rho-\rho^{\prime}\right)^{2}}{4 D \tau}} \mathrm{e}^{-\Delta \rho^{\prime}}+\int_{\rho-\rho_{s 0}}^{\infty} \mathrm{d} \rho^{\prime} \mathrm{e}^{-\Delta \rho^{\prime}} .
$$

As in Sect. 2, this representation is valid only in the regime where the amplitude is weak, $T(\rho, \tau) \ll 1$, but it can be used to approach the unitarity limit $T \sim 1$, and thus compute the dilaton saturation momentum. The first integral in the r.h.s. of Eq. (3.16) involves the dilaton components living at relatively small values of $u$, which scatter only weakly off the incoming $\mathcal{R}$-boson. The second integral refers to the components at larger values of $u$, which undergo strong scattering, but are exponentially suppressed by the dilaton wavefunction. (Of course, when $\tau \leq \tau_{\text {cr }}$ only the first integral would be present.) Accordingly, the overall amplitude is dominated by the components living near the infrared cutoff, so like in pQCD.

Indeed, the integrand in the first integral is peaked at $\rho^{\prime}=\rho-2 \Delta D \tau$; hence, for $\rho<2 \Delta D \tau$, that integral is dominated by its lower limit $\rho^{\prime}=0$, which yields

$$
T(\rho, \tau) \sim \frac{1}{N_{c}^{2}} \mathrm{e}^{\omega_{0} \tau} \mathrm{e}^{-\frac{\rho^{2}}{4 D \tau}}+\frac{1}{\Delta} \mathrm{e}^{-\Delta\left(\rho-\rho_{s 0}\right)} .
$$

This amplitude becomes of $\mathcal{O}(1)$ when $\rho \simeq \rho_{s 0}(\tau)$, meaning that the saturation momentum of the dilaton coincides with that of its component located at $u \simeq u_{0}: \rho_{s}(\tau)=\rho_{s 0}(\tau)$. However, the above calculation of $\rho_{s}$ is consistent only so long as $\rho_{s}<2 \Delta D \tau$, meaning $\rho_{s 0}<2 \Delta D \tau$, which in turn requires $\tau<\tau_{d}$, with

$$
\tau_{d} \equiv \frac{\ln N_{c}^{2}}{\omega_{0}-D \Delta^{2}}=\frac{\ln N_{c}^{2}}{1-(2 / \sqrt{\lambda})\left(\Delta^{2}+1\right)}
$$

When $\tau<\tau_{d}$, one can check that the second term in Eq. (3.17) is exponentially suppressed next to the first one, and therefore

$$
T(\rho, \tau) \simeq \frac{1}{N_{c}^{2}} \mathrm{e}^{\omega_{0} \tau} \mathrm{e}^{-\frac{\rho^{2}}{4 D \tau}} \quad \text { when } \quad \tau<\tau_{d} \quad \text { and } \quad \rho<2 \Delta D \tau
$$

Thus, in this regime, the scattering is controlled by the component of the dilaton living near the IR cutoff $u_{0}=\Lambda R^{2}$, in full analogy to what happens in pQCD (recall the discussion after Eq. (2.26)). In particular, within the restricted interval $\tau_{\text {cr }}<\tau<\tau_{d}$, Eq. (3.19) can be rewritten in a form which resembles the expansion of the QCD amplitude near the saturation line (compare to Eq. (2.27)

$$
T(\rho, \tau) \simeq \mathrm{e}^{-(1-\gamma)\left(\rho-\rho_{s}\right)} \mathrm{e}^{-\frac{\left(\rho-\rho_{s}\right)^{2}}{4 D \tau}} \quad \text { when } \quad \tau_{\text {cr }}<\tau<\tau_{d} \quad \text { and } \quad \rho_{s}<\rho<2 \Delta D \tau,
$$


with the 'anomalous dimension' $\gamma(\tau)$ given by

$$
1-\gamma(\tau)=\frac{\rho_{s 0}(\tau)}{2 D \tau}=\sqrt{\frac{\omega_{0} \tau-\ln N_{c}^{2}}{D \tau}}
$$

Note however that, even for $\rho$ close to $\rho_{s}$, where the diffusion term can be ignored in Eq. (3.20), this amplitude does not show geometric scaling (unlike in pQCD), because the $\tau$-dependence of the 'anomalous dimension' (3.21) cannot be neglected within the corresponding validity range. This difference can be understood as follows: in pQCD, expressions like Eq. (2.27) are valid up to arbitrarily large values of $\tau$, much larger than the critical value $\tau_{\text {cr }} \sim\left(1 / \bar{\alpha}_{s}\right) \ln \left(1 / \alpha_{s}^{2}\right)$ for the onset of saturation; then properties like geometric scaling emerge via asymptotic expansions valid at $\tau \gg \tau_{\text {cr }}$. By contrast, in the present context, Eq. (3.20) is valid only within a limited range of values for $\tau$ above $\tau_{\text {cr }}$, where such asymptotic expansions are not possible anymore.

The case where $\rho>2 \Delta D \tau$ can be similarly treated. For $\tau<\tau_{d}$, the saddle point $\rho^{\prime}=$ $\rho-2 \Delta D \tau$ lies within the integration range and gives the dominant contribution to the overall amplitude:

$$
T(\rho, \tau) \simeq \frac{1}{N_{c}^{2}} \mathrm{e}^{\left(\omega_{0}+D \Delta^{2}\right) \tau-\Delta \rho} \quad \text { when } \quad \tau<\tau_{d} \quad \text { and } \quad \rho>2 \Delta D \tau
$$

in agreement with a corresponding result in Ref. [15]. For $\tau>\tau_{d}$, Eq. (3.16) would predict $T(\rho, \tau) \sim \mathrm{e}^{-\Delta\left(\rho-\rho_{s 0}\right)}$, but this result cannot be trusted anymore since, as we shall shortly argue, Eq. (3.16) is not a reliable approximation for such large values of $\rho$ and $\tau$.

To see that, consider the prediction of Eq. (3.16) for the sum-rule (3.14). When computing the integral there, the amplitude (3.16) can be replaced by its simpler expression in Eq. (3.19) (this will be checked later). To also account for the unitarity bound, we shall write

$$
T(\rho, \tau) \simeq \begin{cases}\frac{1}{N_{c}^{2}} \mathrm{e}^{\omega_{0} \tau} \mathrm{e}^{-\frac{\rho^{2}}{4 D \tau}} & \text { for } \tau<\tau_{s}(\rho) \\ 1 & \text { for } \tau \gtrsim \tau_{s}(\rho) .\end{cases}
$$

Here, $\tau_{s}(\rho)$ is the dilaton saturation line expressed as a function of $\rho$ (the 'inverse' of the function $\left.\rho_{s}(\tau)\right)$; that is, $\tau_{s}(\rho)$ is the rapidity at which the unitarity corrections become important on the resolution scale fixed by $\rho$. Using Eq. (3.13), one immediately finds

$$
\tau_{s}(\rho)=\frac{\ln N_{c}^{2}}{2 \omega_{0}}+\sqrt{\left(\frac{\ln N_{c}^{2}}{2 \omega_{0}}\right)^{2}+\frac{\rho^{2}}{4 D \omega_{0}}} .
$$

After using (3.23) together with the estimate (3.15) for $F_{2}$, the integral in Eq. (3.14) becomes

$$
M(\rho) \sim \mathrm{e}^{\rho} \int_{0}^{\tau_{s}} \mathrm{~d} \tau \mathrm{e}^{-\left(1-\omega_{0}\right) \tau} \mathrm{e}^{-\frac{\rho^{2}}{4 D \tau}}+N_{c}^{2} \mathrm{e}^{\rho} \int_{\tau_{s}}^{\infty} \mathrm{d} \tau \mathrm{e}^{-\tau} \equiv M_{1}+M_{2}
$$

The second integral is trivially performed, and its result is found to rapidly vanish at large $\rho$ :

$$
M_{2}(\rho) \sim N_{c}^{2} \mathrm{e}^{\rho} \mathrm{e}^{-\tau_{s}(\rho)} \longrightarrow \exp \left\{-\frac{\lambda^{1 / 4}}{\sqrt{8}} \rho\right\} \text { for } \rho \rightarrow \infty
$$


Hence, the only way to satisfy the sum-rule would be via the first integral, $M_{1}$. The corresponding integrand is strongly peaked near the saddle point at

$$
\tau^{*}(\rho)=\frac{\sqrt{\lambda}}{4} \rho
$$

Note that, for $\tau \sim \tau^{*}$, we have $2 \Delta D \tau \sim \Delta \rho>\rho$, which justifies our use of the simplified expression (3.19) for the single-Pomeron amplitude in Eq. (3.16).

So long as $\tau^{*}(\rho)<\tau_{s}(\rho)$, this saddle point lies inside the integration domain and can be used to estimate the integral. The ensuing result for $M_{1}(\rho)$ is independent of $\rho$, showing that the sum-rule is saturated by the single-Pomeron exchange, as it should for this to be a consistent approximation. This is the situation considered in Ref. [15], within the framework of the strict large $-N_{c}$ limit. Note, however, that in that limit, the saturation rapidity $\tau_{s}$ was effectively pushed up to infinity and unitarity has never been an issue ${ }^{3}$.

However, when $N_{c}$ is kept finite (although large), unitarity is an issue, which modifies, in particular, the previous calculation of $M_{1}(\rho)$ at large values of $\rho$. Namely, the function $\tau^{*}(\rho)$ grows faster than $\tau_{s}(\rho)$, so the two curves cross each other at a point (see also Fig. 3)

$$
\left(\tau_{c}, \rho_{c}\right)=\left(\frac{\ln N_{c}^{2}}{1-4 / \sqrt{\lambda}}, \frac{4 \ln N_{c}^{2}}{\sqrt{\lambda}-4}\right)
$$

Hence, when $\rho>\rho_{c}$, the saddle point (3.27) lies outside the integration range. Then, the integral giving $M_{1}$ is dominated by its upper cutoff at $\tau=\tau_{s}(\rho)$, which however yields only a tiny result, of the same order as $M_{2}$ (since for $\tau \sim \tau_{s}(\rho)$, the amplitude (3.23) is $\mathcal{O}(1)$ ).

Thus, for $\rho>\rho_{c}$ the sum-rule is not saturated by the single Pomeron exchange anymore, meaning that some new physical mechanism, which was missed by this approximation, should become important in this region. The above calculation gives us some indications in that respect: We have already noticed that, when increasing $\rho$ towards $\rho_{c}$, the saddle point (3.27) approaches the saturation line (3.24), that it crosses exactly at $\rho=\rho_{c}$. It is then reasonable to assume that, for $\rho>\rho_{c}$, the saddle point should remain along the saturation line: $\tau^{*}(\rho)=\tau_{s}(\rho)$. (One cannot have $\tau^{*}(\rho)>\tau_{s}(\rho)$, since the integral over $\tau$ in the saturation region is always dominated by its lower limit $\tau_{s}(\rho)$, as manifest on Eq. (3.25).) If so, then the sum-rule at large $\rho$ can be estimated as in Eq. (3.26): $M(\rho) \sim N_{c}^{2} \exp \left\{\rho-\tau_{s}(\rho)\right\}$. This estimate, together with the condition $M(\rho)=\mathcal{O}(1)$, allows us to predict the form of the saturation line at large $\rho>\rho_{c}$ :

$$
\tau_{s}(\rho) \simeq \rho+\ln N_{c}^{2}, \quad \text { or } \quad \rho_{s}(\tau) \simeq \tau-\ln N_{c}^{2} .
$$

This discussion suggests that the complete saturation line should actually involve two branches, given by Eq. (3.24) for $\rho<\rho_{c}$ and, respectively, Eq. (3.29) for $\rho>\rho_{c}$. Remarkably, these two branches appear to continuously match with each other at the transition point (3.28) : both their values and their first derivatives coincide at this point (see Fig. 3).

What should be the unitarization mechanism responsible for the saturation line in Eq. (3.29)? Once again, the previous sum-rule calculation gives us an indication in that sense: Along the

\footnotetext{
${ }^{3}$ This is also the situation in pQCD, where the sum-rule (3.14) is dominated by relatively large values of $x \sim \mathcal{O}(1)$, hence its calculation is insensitive to unitarity corrections, or even to the BFKL evolution.
} 
line which controls the sum-rule, the energy dependence of the $\mathcal{R}$-boson-dilaton amplitude is precisely that of the massless graviton (not Pomeron) exchange:

$$
\mathrm{e}^{\omega_{0} \tau} \mathrm{e}^{\frac{-\rho^{2}}{4 D \tau}}=\mathrm{e}^{(1-4 / \sqrt{\lambda}) \tau}=\mathrm{e}^{\tau} \mathrm{e}^{-\rho} .
$$

(We have repeatedly used $\tau=(\sqrt{\lambda} / 4) \rho$, cf. Eq. (3.27).) Eq. (3.30) is indeed the same as the propagator of a massless graviton. This result is quite suggestive : The shift of the Pomeron intercept $1 \rightarrow \omega_{0}$ in Eq. (3.9) can be understood as a result of gravitons acquiring a mass due to the curvature of $A d S_{5}$ (see Sect. 4.2 below for details). However, along the line Eq. (3.27), and in fact in the whole region on the right of this line as we shall see, the presence of massless gravitons is inevitable. The graviton exchange is dominantly real and much more non-local than the Pomeron exchange, which is non-local only due to diffusion. However, double, or multiple, graviton exchanges can generate imaginary parts, via the diffractive final states alluded to at the beginning of this subsection. As we shall explicitly see later on, such multiple exchanges are indeed responsible for unitarization at $\rho>\rho_{c}$ and they produce the saturation line in Eq. (3.29).

\section{Mapping the high-energy 'phase diagram'}

Motivated by the above considerations, in this section we shall study the Pomeron propagator in the $A d S_{5}$ geometry, with due attention to both its real and its imaginary part. This will allow us to follow the transition between a genuine Pomeron behaviour at relatively low values of $\rho$, where the curvature effects are important and give rise to an imaginary part and to diffusion, and a massless graviton propagator at large $\rho$, which is long-ranged and predominantly real. Then we shall explain how multiple graviton exchanges generate an imaginary part via diffractive processes, and compute the corresponding saturation line. As a preliminary, we shall make some comments on the anomalous dimension of twist-two operators at strong coupling, which justify the use of the 'diffusion approximation' in the approach towards the saturation line.

\subsection{Anomalous dimension of twist-two operators in $\mathcal{N}=4 \mathrm{SYM}$}

The variable $\gamma$ introduced in Sect. 2 has the interpretation as the anomalous dimension of the twist-two, spin- $j$ gluonic operator

$$
\operatorname{Tr}\left[F_{\mu}^{+}\left(D^{+}\right)^{j-2} F^{+\mu}\right]
$$

with $j$ analytically continued to non-integer values. (In the BFKL context one has $j=1+\chi(\gamma)$; see [37] for discussions on the anomalous dimension and the operator product expansion in that framework.) Recall that the total dimension $\Delta(j)$ of the operator Eq. (4.1) is given by ${ }^{4}$

$$
\Delta(j)=j+2-2 \gamma(j)
$$

In the weak coupling expansion, $\gamma \sim \mathcal{O}\left(\alpha_{s}\right)$, while the BFKL resummation gives a number of $\mathcal{O}(1)$ (and comprised between 0 and $1 / 2$; cf. Sect. 2). On the other hand, for $\mathcal{N}=4$ SYM at

\footnotetext{
${ }^{4}$ This is the common definition of $\gamma$ in the small- $x$ literature, which differs by a factor -2 from the one which is most often used in the literature. A yet different convention $\gamma \rightarrow 1-\gamma$ is sometimes used.
} 
strong 't Hooft coupling $\lambda=g^{2} N_{c} \gg 1$, the AdS/CFT correspondence predicts that the twisttwo operators with $j \neq 2$ receive a large, negative (in our conventions), anomalous dimension. Specifically, Ref. [39] has found

$$
\gamma(j) \approx-\frac{\sqrt{\lambda}}{2 \pi} \ln \frac{j}{\sqrt{\lambda}},
$$

for $j \gg \sqrt{\lambda}$ and, respectively,

$$
\gamma(j) \approx-\sqrt{\frac{j}{2}} \lambda^{\frac{1}{4}}
$$

for $1 \ll j \ll \sqrt{\lambda}$. These results were obtained by computing the energy and the angular momentum of a 'folded' closed string state rotating in the $A d S_{5}$ space. When $j \ll \sqrt{\lambda}$, the string is spinning in a small region compared with the radius $R$, while the case $j \gg \sqrt{\lambda}$ corresponds to a macroscopic string stretched over a distance larger than $R$.

Because of the large anomalous dimension for twist-two operators (excepting the energy momentum tensor which has zero anomalous dimension $\gamma(2)=0$ ), it has been argued in [15] that the OPE in DIS at strong coupling must be considerably reorganized. The dominant contribution comes from the 'protected' (i.e., non-renormalized), double-trace, operators which create and annihilate the entire hadron of interest. These operators are nominally higher-twist, but since their dimension is of order unity, their contribution is less suppressed compared to the twist-two operators having $|\gamma(j)| \gg 1$ for generic $j \neq 2$. Hence DIS at moderate values of $x$ can be pictured as a process where a virtual photon knocks off an entire charged hadron - a drastically different picture from pQCD where a photon knocks off partons inside the hadron.

While plausible in the Bjorken limit $\left(Q^{2} \rightarrow \infty\right.$ at fixed $\left.\tau\right)$, this is no longer the case in the Regge limit $\left(\tau \rightarrow \infty\right.$ at fixed $\left.Q^{2}\right)$. The important regime at high energy is $j \sim 2$. Since $\gamma(2)=0$ exactly, $\gamma(j)$ remains small in the vicinity of $j=2$, and the twist-two operators (analytically continued to complex values of $j$ near $j=2$ ) should still give the leading contribution. Notably, Refs. [38] and [16] arrived at the same expression for $\gamma(j)$ in the 'diffusion approximation' from very different arguments. It is obtained as the solution to

$$
j-j_{0}=\frac{1}{2 \sqrt{\lambda}}(\Delta-2)^{2}=\frac{1}{2 \sqrt{\lambda}}(j-2 \gamma(j))^{2},
$$

where

$$
j_{0}=2-\frac{2}{\sqrt{\lambda}}=1+\omega_{0}
$$

is the Pomeron intercept. Notice that the property $\gamma(2)=0$ is indeed verified by the solution to Eq. (4.5). Some light on the origin of Eq. (4.5) will be shed by the manipulations in the next subsection. The inverse function $j(\gamma)$ is approximately quadratic in $\gamma$ and has a minimum at

$$
\gamma_{0}=1-\frac{1}{\sqrt{\lambda}}
$$

with the minimum value $j\left(\gamma_{0}\right)=j_{0}$. 
In general, the diffusion approximation is expected to be valid only in the vicinity of this minimum, i.e., when $\gamma \sim \gamma_{0}, j \sim j_{0}$. Away from $j=2$, one could a priori expect significant nonlinear corrections to the right hand side of Eq. (4.5). This is precisely what happens at weak coupling, in the context of the BFKL equation, where the diffusion approximation corresponding to $\gamma_{0}=1 / 2$, cf. Eq. 2.14 , significantly deviates from the exact BFKL characteristic function already when $\gamma=\gamma_{s} \simeq 0.37$. (This is why, in that context, it would be incorrect to compute the saturation line by 'unitarizing' the amplitude (2.15) obtained via the diffusion approximation).

However, it turns out that at strong coupling the diffusion approximation is much more robust and has a much wider validity range than at weak coupling. For instance, one can see that Eq. (4.5) correctly reproduces the leading behavior, Eq. (4.4), for large (but not asymptotically large) $j$. (As a matter of fact, Ref. [38] has determined the proportionality constant in Eq. (4.5) in such a way to match Eq. (4.4). But the derivation of Ref. [16] does not rely on this matching.) Moreover, Eq. (4.5) coincides with the well-known anomalous dimension formula of a scalar field in $A d S_{5}$

$$
\Delta=2+\sqrt{4+m^{2} R^{2}}
$$

where $m$ is the mass of the state lying on the leading Regge trajectory (i.e., the highest spin state in a given excitation level $n=0,1,2, \ldots)$ :

$$
m^{2}=\frac{4 n}{\alpha^{\prime}}=\frac{2(j-2)}{\alpha^{\prime}} .
$$

This shows that, in this strong-coupling context, the quadratic relation $j \sim \gamma^{2}$ ('diffusion approximation') has its roots in the Regge behavior of string excited states $\Delta^{2} \sim m^{2} \sim j$, which is expected to hold in $A d S_{5}$ for generic values of $j$ up to $j \sim \sqrt{\lambda}$ (see Eq. (4.4)). This range is large enough to cover all values of $j$ and $\gamma$ of interest at high energy, as we shall later verify.

\subsection{The Pomeron propagator}

In Eq. (3.5), the Virasoro-Shapiro formula in flat space was used rather heuristically [15]. For a more coherent discussion on both the real and the imaginary parts of the amplitude, it is important to carefully derive this amplitude from the fundamental $S$-matrix in string theory, starting with the operator representation of the latter in curved spacetime. Up to a factor $g_{s}^{2}$ to be introduced in the final result, this representation reads [23, 16]

$$
\begin{aligned}
\int \mathrm{d}^{2} z\langle\mathcal{V}(\infty) \mathcal{V}(1) \mathcal{W}(z) \mathcal{W}(0)\rangle \approx \int_{|z|<1} \mathrm{~d}^{2} z\left\langle\mathcal{V}(\infty) \mathcal{V}(1) z^{L_{0}-2} \bar{z}^{L_{0}-2} \mathcal{W}(1) \mathcal{W}(0)\right\rangle \\
=\left\langle\mathcal{V}(\infty) \mathcal{V}(1) \frac{\delta_{L_{0}, \tilde{L}_{0}}}{L_{0}+\tilde{L}_{0}-2} \mathcal{W}(1) \mathcal{W}(0)\right\rangle .
\end{aligned}
$$

Here, $\mathcal{V}$ 's and $\mathcal{W}$ 's are the vertex operators corresponding to dilatons and, respectively, gauge bosons in the $(0,0)$ picture, and the integration is over the string world-sheet with spherical topology. The average is understood in the sense of the string path integral, which involves an integral over the zero modes and a path integral over the nonzero ones. Furthermore, $L_{0}$ and $\tilde{L}_{0}$ are the right- and left-moving Virasoro operators which generate the scale transformations on the world-sheet. The kernel $\delta_{L_{0}, \tilde{L_{0}}} /\left(L_{0}+\tilde{L}_{0}-2\right)$ in the last expression can be recognized as the propagator of the closed string exchanged in the $t$-channel. 
One can diagonalize the operators $L_{0}$ and $\tilde{L}_{0}$ by inserting a complete set of string states the 'Pomeron states' - , which are the states exchanged in the $t$-channel at high-energy [16]. To that aim, one inserts the identity as a sum over the respective projection operators

$$
1=\sum_{j} \int \frac{\mathrm{d} \nu}{2 \pi}\left|\mathcal{V}_{j,-\nu}^{-}\right\rangle\left\langle\mathcal{V}_{j, \nu}^{+}\left|\longrightarrow \int_{C} \frac{\mathrm{d} j}{2 i} \frac{1+\mathrm{e}^{-i \pi j}}{\sin \pi j} \int \frac{\mathrm{d} \nu}{2 \pi}\right| \mathcal{V}_{j,-\nu}^{-}\right\rangle\left\langle\mathcal{V}_{j, \nu}^{+}\right|,
$$

where the sum runs over all the positive, even, values of the spin : $j=2,4, \ldots$, corresponding to the string states on the leading Regge trajectory in Eq. (4.9). Alternatively, the sum over $j$ can be realized as an integration in the complex $j$-plane, as indicated in the r.h.s.; the contour $C$ encircles the positive part of the real axis and is conveniently deformed into a contour $L$ which runs parallel to the imaginary axis, on the left of all the poles at $j>0$ (see Fig. 2 below). (More precisely, this contour deformation within the Sommerfeld-Watson representation is truly an analytic continuation from the $t$-channel amplitude related to the physical, $s$-channel, amplitude via crossing symmetry $(t \leftrightarrow s)$; see, e.g., 40].)

In the equation above, $\mathcal{V}^{ \pm}$is the 'Pomeron vertex operator' [16] which is dual to the twisttwo operator, Eq. (4.1), of the gauge theory (see also [41]). For large $u$, it takes the form

$$
\mathcal{V}_{j, \nu}^{ \pm} \sim U^{j-2-2 i \nu}\left(\partial_{z} X^{ \pm} \partial_{\bar{z}} X^{ \pm}\right)^{\frac{j}{2}}
$$

Capital letters denote world-sheet fields, while lower case letters refer to their zero modes. Also, light-coordinates are defined in the standard way: $X^{ \pm}=\left(X^{0} \pm X^{3}\right) / \sqrt{2}$, with the collision axis taken along $X^{3}$. For what follows it is useful to notice that the vertex operators associated with the dilaton $(\mathcal{V})$ and the $\mathcal{R}$-boson $(\mathcal{W})$ admit representations similar to that in Eq. (4.12), but with the $U$-factor replaced by the corresponding wavefunction $-\Psi(U)$ in the case of the dilaton and, respectively, $A_{m}(U)$ for the $\mathcal{R}$-boson.

The operator (4.12) has total dimension $\Delta=2+2 i \nu$; together with Eq. (4.2), this implies the following anomalous dimension :

$$
\gamma=\frac{j}{2}-i \nu
$$

which appears to be different from the one usually associated with a leading-twist operator in the diffusion approximation, cf. Eq. (2.13). Let us open here a parenthesis to explain this discrepancy: in the leading logarithmic approximation used throughout Sect. 2, the reference scale of the logarithm $\tau=\ln s$ is ambiguous and usually taken to be a product of the two external scales. This ambiguity is resolved in the next-to-leading log approximation (NLLA) where $\gamma$ is shifted in the following way [2]

$$
\left(\frac{s}{Q \Lambda}\right)^{j-1}\left(\frac{Q^{2}}{\Lambda^{2}}\right)^{\frac{1}{2}-i \nu}=\left(\frac{s}{Q^{2}}\right)^{j-1}\left(\frac{Q^{2}}{\Lambda^{2}}\right)^{\frac{j}{2}-i \nu}
$$

to ensure that the Pomeron behaviour $\left(s / Q^{2}\right)^{j-1}$ matches with the definition of the Bjorken-x variable in DIS (at high energy).

The action of $L_{0}$ on the Pomeron vertex operator has been evaluated for $j \approx 2$ [16] as

$$
L_{0} \mathcal{V}_{j, \nu}^{ \pm}=\tilde{L}_{0} \mathcal{V}_{j, \nu}^{ \pm}=\left(\frac{j}{2}-\frac{\alpha}{4} \Delta_{j}^{2}\right) \mathcal{V}_{j, \nu}^{ \pm}=\left(\frac{j}{2}+\frac{\nu^{2}+1}{\sqrt{\lambda}}\right) \mathcal{V}_{j, \nu}^{ \pm},
$$


where (c.f. Eq. (3.8))

$$
\Delta_{j} \equiv\left(\frac{U}{R}\right)^{j} \nabla_{0}^{2}\left(\frac{U}{R}\right)^{-j}
$$

The $\nu$-integral (or the $j$-integral) sets $L_{0}=\tilde{L_{0}}=1$, which after also using Eqs. (4.13)-(4.15) is recognized as the previous condition (4.5). So, Eq. (4.5) simply states that $\mathcal{V}^{ \pm}$is a $(1,1)$ tensor on the world-sheet in the curved background of $A d S_{5}$ [42].

We are now prepared to evaluate the string amplitude in Eq. (4.10) in the semi-classical approximation. After using Eqs. (4.11) and (4.15), one finds

$$
\begin{aligned}
& \left\langle\mathcal{V}(\infty) \mathcal{V}(1) \frac{\delta_{L_{0}, \tilde{L}_{0}}}{L_{0}+\tilde{L}_{0}-2} \mathcal{W}(1) \mathcal{W}(0)\right\rangle= \\
& =\int \frac{\mathrm{d} j \mathrm{~d} \nu}{4 \pi i} \frac{1+\mathrm{e}^{-i \pi j}}{\sin \pi j} \frac{1}{j-j_{0}+\frac{2 \nu^{2}}{\sqrt{\lambda}}}\left\langle\mathcal{V}(\infty) \mathcal{V}(1) \mathcal{V}_{j,-\nu}^{-}\right\rangle\left\langle\mathcal{V}_{j, \nu}^{+} \mathcal{W}(1) \mathcal{W}(0)\right\rangle
\end{aligned}
$$

When evaluating expectation values in the semi-classical limit, one can replace an operator $F(U)$ on the world-sheet with its value $F(u)$ at position $u$ (the zero mode $U$ ). Besides, the path integral over $U$ reduces to the ordinary integral over $u$, with the appropriate, invariant, measure. Since the string propagator in Eq. (4.17) is non-local in $u$, some care is needed when constructing the measure. Namely, in the decomposition of unity, Eq. (4.11), we need to insert the vertex operators at different positions $u$ and $u^{\prime}$. The appropriate decomposition reads then

$$
1=\int_{L} \frac{\mathrm{d} j}{2 i} \frac{1+\mathrm{e}^{-i \pi j}}{\sin \pi j} \int \frac{\mathrm{d} \nu}{2 \pi} \int \frac{\mathrm{d} u^{\prime}}{R} \sqrt{-G^{\prime}}\left(G^{\prime+-}\right)^{j}\left|\mathcal{V}_{j,-\nu}^{-}\left(u^{\prime}\right)\right\rangle\left\langle\mathcal{V}_{j, \nu}^{+}(u)\right|,
$$

where $G$ is the determinant of the $A d S_{5}$ metric $G_{m n}$, and $G^{+-}$is the respective metric component; Eq. (3.1) implies $G^{+-}=R^{2} / u^{2}$ and $\sqrt{-G}=(u / R)^{3}$. Eq. (4.18) follows from the following decomposition of the $\delta$-function, which can be easily checked:

$$
1=\int \mathrm{d} u^{\prime} \delta\left(u-u^{\prime}\right)=\int \frac{\mathrm{d} u^{\prime}}{R} \sqrt{-G^{\prime}}\left(G^{\prime+-}\right)^{j} \int \frac{\mathrm{d} \nu}{2 \pi}\left(\frac{u^{\prime}}{R}\right)^{j-2+2 i \nu}\left(\frac{u}{R}\right)^{j-2-2 i \nu} .
$$

One can now insert the explicit expressions for the vertex operators in the $u$-representation and then perform the integral over $\nu$ by deforming the contour in the complex plane. Using the fact that $u>u^{\prime}$ in DIS at large $Q^{2}$ (recall that $u \sim Q$, whereas $u^{\prime} \sim u_{0}$ ), we shall close the contour in the lower half plane, and thus pick up the pole at

$$
\nu(j)=-i \sqrt{\frac{\sqrt{\lambda}}{2}\left(j-j_{0}\right) .}
$$

This gives

$$
\begin{aligned}
& \int \mathrm{d} u \sqrt{-G} \int \frac{\mathrm{d} j}{2 i} \frac{1+\mathrm{e}^{-i \pi j}}{\sin \pi j} \frac{\left(\alpha_{\mathrm{eff}}^{\prime} s\right)^{j}}{\sqrt{j-j_{0}}} \\
& \times \int \mathrm{d} u^{\prime} \sqrt{-G^{\prime}}\left(G^{\prime+-}\right)^{j} \Phi^{\dagger} \Phi\left(u^{\prime}\right) u^{\prime j-2+2 i \nu(j)} u^{j-2-2 i \nu(j)} A_{m}(u, q) A^{m}(u,-q)+\cdots \\
& =\int \frac{\mathrm{d} j}{2 i} \frac{1+\mathrm{e}^{-i \pi j}}{\sin \pi j} \frac{\mathrm{e}^{\tau j}}{\sqrt{j-j_{0}}} \int \frac{\mathrm{d} u}{u} u^{4} \int \frac{\mathrm{d} u^{\prime}}{u^{\prime}} \Phi^{\dagger} \Phi\left(u^{\prime}\right)\left(\frac{u^{\prime 2}}{u^{2}}\right)^{1-\gamma(j)} A_{m}(u, q) A^{m}(u,-q)+\cdots
\end{aligned}
$$


where the dots stay for terms which are subleading at high energy. The factor (cf. Eq. (3.6))

$$
\left(\alpha_{\mathrm{eff}}^{\prime} s\right)^{j}=\left(\frac{\alpha^{\prime} R^{2} s}{u^{2}}\right)^{j} \sim\left(\frac{\alpha^{\prime} R^{2} s}{u_{c}^{2}}\right)^{j}=\left(\frac{1}{\sqrt{\lambda} x}\right)^{j} \sim \mathrm{e}^{\tau j},
$$

arises after contraction with vertex operators which carry large momenta [16]. Apart from the external wavefunctions, Eq. (4.21) is symmetric under the exchange of $u$ and $u^{\prime}$ as it should.

After including the factor $g_{s}^{2} \sim 1 / N_{c}^{2}$ and substituting the solution of the Maxwell equation for $A_{m}(u)$ [15], one can identify the forward scattering amplitude for the current-dilaton scattering, whose imaginary part yields the $F_{2}$ structure function: $F_{2} \propto N_{c}^{2} Q^{2} \operatorname{Im} \tilde{T}$. [We use the notation $\tilde{T}$ for the complete scattering amplitude, including both the real and the imaginary part; hence, $T=\operatorname{Im} \tilde{T}$.] One finds

$$
\begin{aligned}
\tilde{T}\left(x, Q^{2}\right) \simeq & \frac{(Q R)^{6}}{N_{c}^{2}} \int \frac{\mathrm{d} u}{u} \frac{1}{u^{4}}\left(K_{0}^{2}\left(Q R^{2} / u\right)+K_{1}^{2}\left(Q R^{2} / u\right)\right) \\
& \times \int \frac{\mathrm{d} u^{\prime}}{u^{\prime}} \Phi^{\dagger} \Phi\left(u^{\prime}\right) \int \frac{\mathrm{d} j}{2 i} \frac{1+\mathrm{e}^{-i \pi j}}{\sin \pi j} \frac{\mathrm{e}^{\tau(j-1)}}{\sqrt{j-j_{0}}}\left(\frac{u^{\prime 2}}{u^{2}}\right)^{1-\gamma(j)} .
\end{aligned}
$$

Eq. (4.23) exhibits 'radial factorization': it is expressed as a convolution in $u$ of pieces which can be recognized as wavefunctions for the $\mathcal{R}$-boson and, respectively, the dilaton, and a kernel which is by construction the Pomeron propagator in string theory - a sum over all string states exchanged in the $t$-channel at high energy - , and which after multiplication by $g_{s}^{2}$ plays the role of a scattering amplitude between states localized at $u$ and $u^{\prime}$. Clearly, this $u$-factorization is the string counterpart of the ' $k_{T}$-factorization' valid in the high-energy regime at weak coupling (as exhibited, e.g., in Eqs. (2.3) and (2.5)).

Let us concentrate on the elementary Pomeron amplitude, that we would like to compare to its previous approximation in Eq. (3.12). With our usual notation $\rho \equiv \ln \left(u^{2} / u_{0}^{2}\right)$, this can be rewritten as

$$
T_{\mathbb{P}}\left(\rho, \rho^{\prime}, \tau\right)=\frac{1}{N_{c}^{2}} \int_{L} \frac{\mathrm{d} j}{2 i} \frac{1+\mathrm{e}^{-i \pi j}}{\sin \pi j} \frac{\mathrm{e}^{\tau(j-1)}}{\sqrt{j-j_{0}}} \exp \left\{\left(\rho-\rho^{\prime}\right)\left(\frac{j-2}{2}-\sqrt{\frac{\sqrt{\lambda}}{2}\left(j-j_{0}\right)}\right)\right\}
$$

where we have used Eqs. (4.13) and (4.20) to explicit the function $\gamma(j)$. The contour $L$ runs parallel to the imaginary $j$ axis and crosses the real axis between the branch point at $j=j_{0} \equiv$ $2-2 / \sqrt{\lambda}$ and the pole at $j=2$ (see Fig. 2).

For sufficiently high energies, the integral can be evaluated by deforming the contour $L \rightarrow L^{\prime}$, with $L^{\prime}$ encircling the branch cut that ends at $j_{0}$, as shown in Fig. 2. Alternatively, and more conveniently for our present purposes, one can use the saddle point method. Namely, the integrand has a saddle point at

$$
j^{*} \simeq j_{0}+\frac{\sqrt{\lambda}\left(\rho-\rho^{\prime}\right)^{2}}{8 \tau^{2}},
$$

where we have used $\tau \gg \rho-\rho^{\prime}$, as appropriate at high energy. So long as $\sqrt{\lambda}\left(\rho-\rho^{\prime}\right) / 4<\tau$, this saddle point lies between $j_{0}$ and 2 , and then the use of the saddle point approximation is indeed 


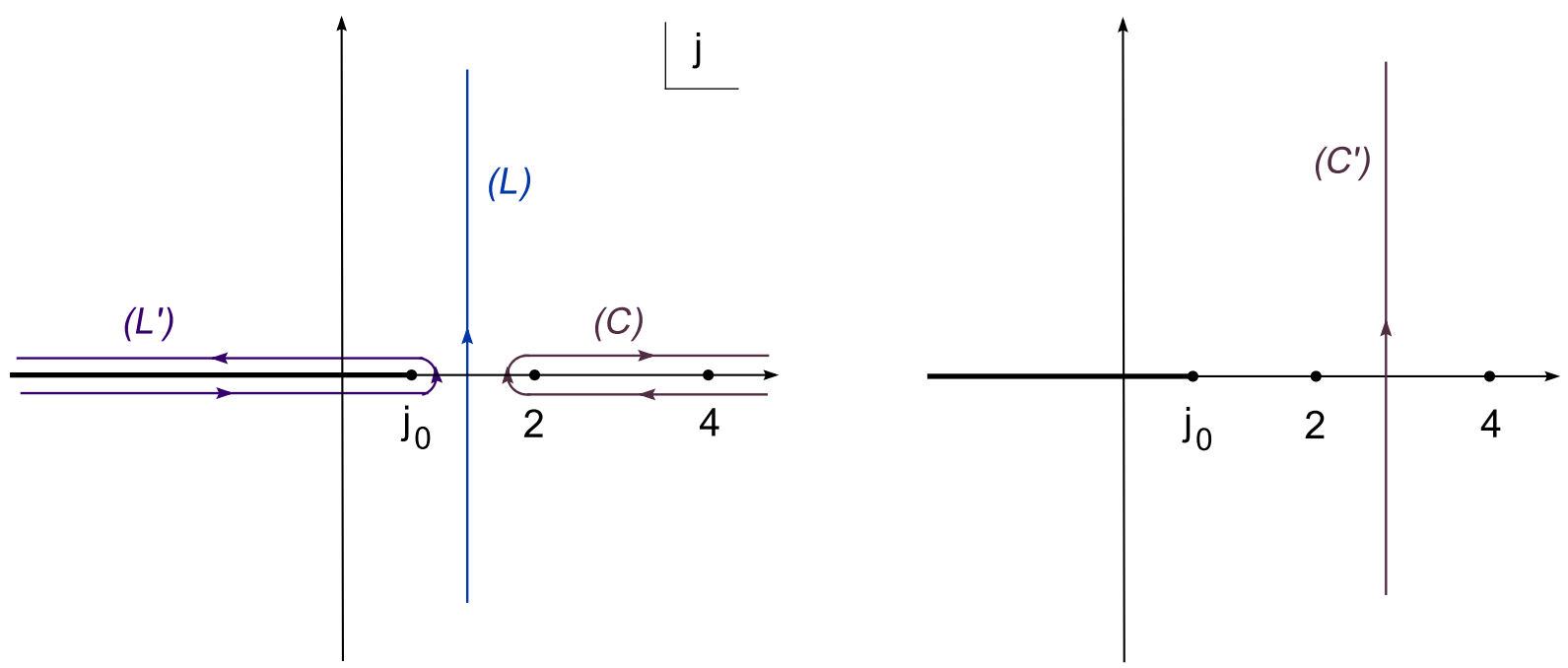

Figure 2: The analytic structure of the integrand in Eq. (4.24) in the complex $j$ plane, together with various contours used when evaluating the integral.

justified. By also using $\mathrm{e}^{-i \pi j_{0}} \approx 1+2 \pi i / \sqrt{\lambda}$, one thus finds that the high-energy amplitude develops an imaginary $\operatorname{part}^{5} T_{\mathbb{P}} \equiv \operatorname{Im} \tilde{T}_{\mathbb{P}}$, with

$$
T_{\mathbb{P}}\left(\rho, \rho^{\prime}, \tau\right) \approx \frac{1}{N_{c}^{2}} \mathrm{e}^{\omega_{0} \tau} \mathrm{e}^{-\left(1-\gamma_{0}\right)\left(\rho-\rho^{\prime}\right)} \mathrm{e}^{-\frac{\left(\rho-\rho^{\prime}\right)^{2}}{4 D \tau}},
$$

where $\omega_{0}=1-2 / \sqrt{\lambda}, \gamma_{0}=1-1 / \sqrt{\lambda}$, and $D=2 / \sqrt{\lambda}$.

Eq. (4.26) coincides with the formula previously used in Sect. 3, cf. Eqs. (3.9) or (3.12), up to a factor $\mathrm{e}^{-\left(1-\gamma_{0}\right) \rho}=\left(u^{\prime 2} / u^{2}\right)^{1 / \sqrt{\lambda}}$. This additional factor has the same origin as the factor $\left(r^{2} / r^{\prime 2}\right)^{1 / 2}$ in the BFKL case, Eq. (2.15) - namely, is reflects the high-energy 'anomalous dimension' - , but it is less important here because the anomalous dimension $\gamma_{0}$ is close to one (in contrast to the BFKL case where $\gamma_{0}=1 / 2$ ). And indeed, by inspection of Eq. (4.26), one can check that the additional term $\left(1-\gamma_{0}\right)\left(\rho-\rho^{\prime}\right)$ in the exponent remains negligible so long as $\rho-\rho^{\prime} \ll \tau$, and hence it cannot affect our previous conclusions in Sect. 3.2. Note also that, in this high-energy approximation, the graviton pole $\sim 1 / t$ is absent in the real part of the string amplitude - in contrast to what happens in flat space - , because of the shift in the Pomeron intercept by the space-time curvature: $\sin \pi j_{0} \neq 0$.

However, the graviton pole reappears when moving to sufficiently large values of $\rho$ (for a given $\tau$ ), as we explain now. Indeed, when

$$
\frac{\sqrt{\lambda}}{4}\left(\rho-\rho^{\prime}\right)>\tau,
$$

the saddle point $j^{*}$ exceeds 2 , and then one has to deform the contour from $C$ to $C^{\prime}$ (see Fig. 2), in order to separately evaluate the pole of $(1 / \sin \pi j)$ at $j=2$. This leads to

$$
\tilde{T}_{\mathbb{P}}\left(\rho, \rho^{\prime}, \tau\right) \approx \frac{\mathrm{e}^{\tau}}{N_{c}^{2}} \mathrm{e}^{-\left(\rho-\rho^{\prime}\right)}+\frac{1}{N_{c}^{2}} \int_{C^{\prime}} \frac{\mathrm{d} j}{2 i} \frac{1+e^{-i \pi j}}{\sin \pi j} \frac{\mathrm{e}^{(j-1) \tau}}{\sqrt{j-j_{0}}} \mathrm{e}^{-(1-\gamma(j))\left(\rho-\rho^{\prime}\right)} .
$$

\footnotetext{
${ }^{5}$ The Virasoro-Shapiro formula in flat space also gives an imaginary part in a similar manner. $\mathrm{e}^{-i \pi \alpha^{\prime} t / 2} / t \approx$ $1 / t-i \pi \alpha^{\prime} / 2$. This is how Eq. (3.5) was actually derived.
} 
The first, purely real, term $\propto \mathrm{e}^{\tau}\left(u^{\prime 2} / u^{2}\right)$ is recognized as the propagator of the elementary, massless, graviton in $A d S_{5}$. For large $\tau$ and $\rho$ (such that the condition (4.27) is satisfied), this pole term dominates over the remaining contour integral. Indeed, the function $f(j) \equiv$ $(j-1) \tau+\gamma(j)\left(\rho-\rho^{\prime}\right)$ obeys $f(2)=\tau, f^{\prime}\left(j^{*}\right)=0$, and $f^{\prime}(j)<0$ for any real $j$ within the interval $2 \leq j<j^{*}$. Hence, whenever (4.27) is satisfied, one can choose the contour $C^{\prime}$ (see Fig. 2) to cross the real axis at some $j_{1}$ with $2<j_{1}<j^{*}$, and then $\operatorname{Re} f(j)<\tau$ for any $j$ along $C^{\prime}$.

Thus for high energy and sufficiently large $\rho$, the 'Pomeron' exchange is dominated by the elementary graviton and the amplitude is predominantly real. Since the graviton propagator is very non-local in $u$, it can connect the large distance between $u \sim Q R^{2}$ and $u^{\prime} \sim \Lambda R^{2}$ without much suppression. Accordingly, with increasing $\tau$, the corresponding amplitude becomes of $\mathcal{O}(1)$ relatively fast (much faster than for the diffusive Pomeron in Eq. (4.26) !), namely when

$$
\tau=\rho-\rho^{\prime}+\ln N_{c}^{2},
$$

in agreement with Eq. (3.29). Of course, being real, this single graviton exchange does not contribute to $F_{2}$. Yet, multiple such exchanges can do so, as we shall shortly explain.

To summarize, the single-Pomeron-exchange approximation to $F_{2}\left(x, Q^{2}\right)$ is expected to be a legitimate approximation at high energy and strong coupling so long as $\rho \equiv \ln Q^{2} / \Lambda^{2}<4 \tau / \sqrt{\lambda}$ and for rapidities $\tau<\tau_{s}(\rho)$, with the saturation rapidity $\tau_{s}(\rho)$ given by Eq. (3.24). The two delimitating curves intersect with each other at the point $\left(\rho_{c}, \tau_{c}\right)$, Eq. (3.28). This is also the point where starts the large $-\rho$ branch of the saturation line, cf. Eqs. (3.29) or (4.29), to be further discussed in the next subsection.

\subsection{Saturation line from multiple graviton exchanges}

The previous analysis shows that the physics of unitarization in DIS at strong coupling and relatively large $Q^{2}$ is conceptually different from what happens either at weak coupling, or at strong coupling but for lower values of $Q^{2}$. In the last two cases, which are the more standard ones, the black disk limit for the scattering amplitude is approached by unitarizing the single Pomeron exchange, which in turn requires energies which are high enough for the two scales involved in the collision to be joint by diffusion. But for strong coupling and large $Q^{2}$, the single Pomeron exchange is unable to saturate the energy-momentum sum rule (3.14) - essentially, because its imaginary part decays too fast at large $Q^{2}$. However, precisely in that large- $Q^{2}$ region, the full Pomeron propagator in string theory develops an additional, real, part, which describes elementary graviton exchanges and has the potential to generate stronger interactions, since very non-local in $u$. This new component can contribute to the DIS structure functions too, but only via multiple scattering. In this subsection, we would like to argue (without performing a fully fledged calculation of multiple scattering in $A d S_{5}$ ) that multiple graviton exchanges dominate indeed at high energy and large $Q^{2}$ (in the vicinity of the saturation line) and, in particular, they saturate the sum rule in the way already suggested after Eq. (3.26).

The presence of a large real part in the Pomeron propagator describing the massless graviton exchange is a common feature in string theories, and hence also in gauge theories with a gravity dual. Multiple scattering in such theories is described by multi-loop diagrams, and at each order of this loop expansion the dominant contribution is provided by the exchange of $n$ gravitons

(for a $(n-1)$-loop amplitude). It is well-known that in flat space such higher loop terms 
exponentiate in the eikonal approximation [43, 12], and the same was recently shown to hold for high energy scattering in $A d S_{5}$ [18, 17]. The eikonal approximation breaks down at very small impact parameters and/or at extremely high energies (see below). In a string dual description of DIS at strong coupling, the impact parameter in the fifth dimension is large $u / u^{\prime} \sim Q / \Lambda$, and therefore the eikonal resummation is expected to work reasonably well.

In general, the exponentiation is done in the three-dimensional impact parameter space spanned by the radial coordinate $u$ and the two-dimensional, physical, impact parameter $\vec{b}$ (neglecting nonlocality on $S^{5}$ ), whereas our previous formulæ, like Eq. (4.24), are already integrated over $\vec{b}$. While it is possible to undo this integral and analyze the three-dimensional propagator following [18, 17], one may anticipate, following the example of QCD at weak coupling (cf. Sect. 2; see also Ref. 44]), that a simplification occurs for relatively central impact parameters, $b \ll 1 / \Lambda$. DIS is characterized by the large resolution scale $Q^{2} \gg \Lambda^{2}$ which determines the size of the 'active' region in the transverse space. Hence, so long as $b \ll 1 / \Lambda$ one can neglect edge effects and work with scattering amplitudes averaged over $b$, so like Eq. (4.24).

In the present context, the eikonal approximation amounts to exponentiating the treelevel 'Pomeron' amplitude Eq. (4.24), which thus becomes the phase shift in the $S$-matrix: $S=\exp \left\{i \tilde{T}_{\mathbb{P}}\right\}$. In the interesting domain at large $\rho \equiv \ln \left(Q^{2} / \Lambda^{2}\right)$, namely, $\rho>4 \tau / \sqrt{\lambda}$, the Pomeron amplitude is given by Eq. (4.28) and has both real and imaginary parts.

The imaginary part describes 'inelastic processes' in which the final states are (generally massive) string excitations, as obtained by cutting through the Pomeron. This imaginary part can be evaluated in the saddle point approximation, with the result displayed in Eq. (4.26). By itself, this contribution would generate the saturation line in Eqs. (3.13) or (3.29), which however has been argued to be inconsistent at large $\rho>\rho_{c}$, in Sect. 3.2.

The relevant contribution at large $\rho$ is rather the one generated by the real part of the phaseshift, that is, by the exponentiation of the elementary graviton exchange. The corresponding imaginary part starts at the level of the two-graviton exchange, where it describes, in particular, the cross-section for the elastic scattering between two states localized at $u$ and $u^{\prime}$, respectively. Note however that, in the context of DIS, this process is not truly elastic - rather, it is a particularly simple diffractive process, which contributes to $F_{2}$-, because the incoming virtual photon (or $\mathcal{R}$-current) does not appear in the final state. The amplitude for such a double graviton exchange is estimated as

$$
T_{2 g}\left(\rho, \rho^{\prime}, \tau\right) \approx\left[\frac{\mathrm{e}^{\tau}}{N_{c}^{2}} \mathrm{e}^{-\left(\rho-\rho^{\prime}\right)}\right]^{2},
$$

and becomes of $\mathcal{O}(1)$ along the saturation curve 4.29). More general diffractive processes characterized by a rapidity gap between the products of the collision — are obtained by 'cutting' the multiple scattering series in between successive graviton exchanges, and by allowing for more general diffractive states: the strings in the final states need not be exactly the same as the incoming ones, rather they can be internal excitations of the latter, like higher Kaluza-Klein modes. Whenever this happens, the process is genuinely absorptive, already at the level of the elementary $\rho-\rho^{\prime}$ amplitude.

The diffractive excitations of the string have been first discussed in Ref. [12] in flat space. Leaving a rigorous treatment of this effect in $A d S_{5}$ for future work, here we only give an estimate of its magnitude. The internal excitation of incoming strings is due to the finiteness of the string 
size. This effect can be understood as the result of the tidal force 445 experienced by one of the strings traversing a gravitational shock wave created by the other. In the operator eikonal approach of [12], the real part of the phase shift reads

$$
\frac{\mathrm{e}^{\tau}}{N_{c}^{2}} \frac{U^{\prime 2}}{U^{2}} \approx \frac{\mathrm{e}^{\tau}}{N_{c}^{2}} \frac{u^{\prime 2}}{u^{2}}+\frac{1}{2} \frac{\partial^{2}}{\partial u^{2}}\left(\frac{\mathrm{e}^{\tau}}{N_{c}^{2}} \frac{u^{\prime 2}}{u^{2}}\right) \tilde{U} \tilde{U}+\cdots
$$

where again capital letters denote operators, decomposed between zero and non-zero modes as $U=u+\tilde{U}$. The non-zero mode $\tilde{U}$ is typically of the order the string size, as measured by a local inertial observer:

$$
\tilde{U} \sim l_{s} \frac{u}{R},
$$

so the expansion parameter in Eq. (4.31) is $l_{s}^{2} / R^{2}=1 / \sqrt{\lambda}$. The strength of string excitations leading to absorption is measured by the second term of Eq. (4.31). It reaches unity when

$$
\tau=\rho-\rho^{\prime}+\ln N_{c}^{2}+\ln \sqrt{\lambda},
$$

which, once again, is parametrically the same as Eq. (4.29). (Remember that we are in the regime where $\ln N_{c}^{2}>\lambda \gg \ln \lambda$.) We thus see that all types of diffractive processes - both the quasi-elastic ones, cf. Eq. (4.30), and the genuinely absorptive ones, cf. Eq. (4.31) - contribute on equal grounds to the imaginary part of the forward scattering amplitude, and approach the unitarity limit along the curve exhibited in Eq. (4.29). This is in contrast to the flat space result [12] where the imaginary part due to string excitations is suppressed with respect to the real part by an inverse power of the impact parameter $l_{s}^{2} / b^{2}$.

So far, the diffractive unitarity line (4.29) has been derived for the scattering between two states localized at $\rho$ and $\rho^{\prime}$. But it is easy to check that a similar line, with $\rho^{\prime}=0$, applies to the dilaton as whole. Indeed, after performing the convolution with the dilaton wavefunction, the relevant amplitude can be evaluated as in Eq. (3.16), which gives

$$
T_{2 g}(\rho, \tau) \simeq \int_{0}^{\rho-\rho_{s 0}} \mathrm{~d} \rho^{\prime}\left[\frac{\mathrm{e}^{\tau}}{N_{c}^{2}} \mathrm{e}^{-\left(\rho-\rho^{\prime}\right)}\right]^{2} \mathrm{e}^{-\Delta \rho^{\prime}}+\int_{\rho-\rho_{s 0}}^{\infty} \mathrm{d} \rho^{\prime} \mathrm{e}^{-\Delta \rho^{\prime}},
$$

where now $\rho_{s 0} \equiv \tau-\ln N_{c}^{2}$, cf. Eq. (4.29), and we have assumed that $\rho>\rho_{s 0}$. In writing the equation above, we have focused on the two-graviton exchange, cf. Eq. (4.30), since this is the dominant contribution when approaching the saturation lien from large values of $\rho$. At this point, it is important to remember that the dilaton conformal dimension satisfies $\Delta>2$, as manifest on Eq. (4.8). Hence, the first integral in Eq. (4.34) is dominated by its lower limit $\rho^{\prime}=0$ and, moreover, the second integral is exponentially suppressed w.r.t. the first one. Thus, once again, we find that the scattering is controlled by the dilaton component living near the infrared cutoff at $u \sim u_{0}$. This yields

$$
T_{2 g}(\rho, \tau) \simeq\left[\frac{\mathrm{e}^{\tau}}{N_{c}^{2}} \mathrm{e}^{-\rho}\right]^{2},
$$

which becomes of $\mathcal{O}(1)$ for $\rho \simeq \rho_{s}(\tau)=\tau-\ln N_{c}^{2}$, in agreement with Eq. (3.29). 
For $\rho>\rho_{c}$, with $\rho_{c}$ defined in Eq. (3.28), the curve (3.29) lies below the unitarity line (3.24) for inelastic processes (see also Fig. 3), meaning that, when increasing $\tau$ in this regime, the unitarization of DIS proceeds via diffractive processes. Therefore, Eq. (3.29) represents the physical saturation line for $\rho>\rho_{c}$. This conclusion is further corroborated by the following facts: (i) at $\rho=\rho_{c}$, the two branches of the saturation line, Eq. (3.24) and, respectively, Eq. (3.29), continuously match with each other, and (ii) for $\rho>\rho_{c}$, the energy-momentum sum rule (3.14) is saturated by the diffractive processes (see below), showing that no important physical mechanism has been left out by the previous analysis.

To evaluate the sum-rule (3.14), notice first that a contribution to $F_{2}$ due to the exchange of $n$ gravitons (with $n \geq 2$ ), is parametrically

$$
F_{2}\left(\tau, Q^{2}\right) \sim N_{c}^{2} \mathrm{e}^{\rho} T_{n g}(\rho, \tau) \sim N_{c}^{2} \frac{Q^{2}}{\Lambda^{2}}\left(\frac{\mathrm{e}^{\tau}}{N_{c}^{2}\left(Q^{2} / \Lambda^{2}\right)}\right)^{n} .
$$

When this is inserted within the Eq. (3.14), it is clear that, for any $n \geq 2$, the integrand is strongly peaked at the saturation value $\tau_{s}=\rho+\ln N_{c}^{2}$ : indeed, the integrand rises exponentially with $\tau$ so long as $\tau<\tau_{s}$, but it is exponentially decreasing at $\tau>\tau_{s}$. Moreover, at the peak value, $T_{n g}\left(\rho, \tau=\tau_{s}\right) \sim \mathcal{O}(1)$, so the integral is evaluated as

$$
\int \mathrm{d} \tau \mathrm{e}^{-\tau} F_{2}\left(\tau, Q^{2}\right) \sim N_{c}^{2} \frac{Q^{2}}{\Lambda^{2}} \mathrm{e}^{-\tau_{s}(\rho)} \sim \mathcal{O}(1) .
$$

This result is independent of $Q^{2}$, as anticipated. Interestingly, the above calculation also shows that, at high $Q^{2}$, most of the total energy (or longitudinal momentum) of the hadron lies along the saturation line (3.29), that is, at relatively large rapidities, or small values of $x$. This is in sharp contrast with what happens in QCD at weak coupling, where the energy of a fast moving hadron is predominantly carried by the hadron constituents ('partons') with relatively large values of $x$, or small values of $\tau$, well below the corresponding saturation value $\tau_{s}(\rho)$. We shall return to this observation in the final, discussion, section, where we shall attempt a partonic interpretation for our results at strong coupling.

We conclude this section with some estimates for $F_{2}\left(\tau, Q^{2}\right)$ in the vicinity of the saturation line (3.29), to be compared to the corresponding results in pQCD, cf. Eqs. (2.30) and (2.31). Note first that Eq. (3.29) implies $Q_{s}^{2}(\tau)=\Lambda^{2}\left(\mathrm{e}^{\tau} / N_{c}^{2}\right)$.

For relatively low virtualities, $Q^{2} \lesssim Q_{s}^{2}(\tau)$, one finds

$$
F_{2}\left(\tau, Q^{2}\right) \sim \frac{N_{c}^{2} Q^{2}}{\Lambda^{2}} \quad \text { when } \quad Q^{2} \lesssim Q_{s}^{2}(\tau)
$$

which shows saturation, in the sense that $F_{2}$ does not increases with $\tau$, and that $F_{2} / Q^{2}$ is not divergent as $Q^{2} \rightarrow 0$. (A physical interpretation for this behaviour will be proposed in Sect. 5.)

For $Q^{2}$ much larger than $Q_{s}^{2}(\tau)$, the structure function is dominated by the two-graviton exchange and scales as

$$
F_{2}\left(\tau, Q^{2}\right) \sim \frac{N_{c}^{2} Q^{2}}{\Lambda^{2}}\left(\frac{\mathrm{e}^{\tau} \Lambda^{2}}{N_{c}^{2} Q^{2}}\right)^{2}
$$

or

$$
\frac{F_{2}\left(\tau, Q^{2}\right)}{Q^{2}} \sim \frac{N_{c}^{2}}{\Lambda^{2}}\left(\frac{Q_{s}^{2}(\tau)}{Q^{2}}\right)^{2} \quad \text { when } \quad Q^{2} \gg Q_{s}^{2}(\tau)
$$


This is geometric scaling (compare to the weak-coupling result in Eq. (2.30), with an effective anomalous dimension $\gamma_{s}=-1$, which however is no longer associated with the anomalous dimension of the twist-two operator. Indeed, the contribution exhibited in Eqs. (4.39) or (4.40) is obviously of higher twist order, and in fact this process has an analog in standard DIS: the 'diffractive dissociation' of the virtual photon, $\gamma^{*} p \rightarrow q \bar{q} p$, which involves the elastic scattering between the 'color dipole' (the $q \bar{q}$ pair) and the proton (see, e.g., [26, 34, 49]). There are however some interesting differences between these results and the corresponding ones at weak coupling:

(i) In pQCD, the diffractive scattering represents only a tiny fraction of the total DIS crosssection, whereas in the present, strong-coupling, case this has been found to be the dominant component at high energy, which in particular is responsible for unitarization.

(ii) The diffractive structure function $F_{2}^{D}$ in pQCD at high energy (small $x$ ) and large $Q^{2}$ is formally a leading-twist quantity, $F_{2}^{D} / Q^{2} \sim 1 / Q^{2}$, although the relevant dipole amplitude is, of course, of higher-twist order. This is so since, in computing $F_{2}^{D}$, the convolution with the virtual photon wavefunction, Eq. (2.4), is dominated by the relatively large $q \bar{q}$ excitations having $r \gg 1 / Q$ (the so-called 'aligned-jet configurations') [34, 49]. This does not happen, however, in the present context at strong coupling, where the convolution in Eq. (4.23) is controlled by $u \sim u_{c} \sim Q$. Accordingly, the ensuing structure function is a higher-twist quantity, cf. Eq. (4.40), so like the elementary scattering amplitude in Eq. (4.30).

\section{Physical discussion: Towards a partonic picture at strong coupling}

Let us first summarize the main new results in this paper, before we propose a physical interpretation for them. (These results are also graphically summarized in Fig. 3.) By studying deep inelastic scattering at strong 't Hooft coupling and high energy within the dual string theory, we have inferred the position of the saturation line which separates the weak-scattering regime from the strong-scattering one in the kinematical plane $\tau-\rho$, with $\tau=\ln \left(s / Q^{2}\right)$ and $\rho=\ln \left(Q^{2} / \Lambda^{2}\right)$. This line is in fact the juxtaposition of two curves, matching with each other at the point $\left(\rho_{c}, \tau_{c}\right)$ defined in Eq. (3.28), which correspond to the two different mechanisms responsible for unitarization in different kinematical regions :

- For $\rho \leq \rho_{c} \simeq 4 \ln N_{c}^{2} / \sqrt{\lambda}$, the scattering amplitude in the vicinity of the unitarity limit is controlled by a single Pomeron exchange; this amplitude becomes of $\mathcal{O}(1)$ on the saturation line given by Eqs. (3.13) or (3.24), i.e., when $Q^{2}=Q_{s}^{2}(\tau)$, with the saturation momentum

$$
Q_{s}^{2}(\tau)=\Lambda^{2} \mathrm{e}^{\sqrt{4 D \tau\left(\omega_{0} \tau-\ln N_{c}^{2}\right)}} \quad \text { for } \quad \tau_{\text {cr }}<\tau<\tau_{c},
$$

with $\omega_{0}=1-2 / \sqrt{\lambda}, D=2 / \sqrt{\lambda}$, and $\tau_{\text {cr }} \simeq \ln N_{c}^{2}$ is the critical rapidity for the onset of unitarity corrections.

- For $\rho>\rho_{c}$, the dominant contribution to the DIS structure function before unitarization comes from multiple exchanges of elementary gravitons; the reaches the unitarity limit on the saturation line shown in Eq. (3.24), i.e., for $Q^{2}=Q_{s}^{2}(\tau)$, with

$$
Q_{s}^{2}(\tau)=\Lambda^{2} \frac{\mathrm{e}^{\tau}}{N_{c}^{2}} \quad \text { for } \quad \tau>\tau_{c}
$$




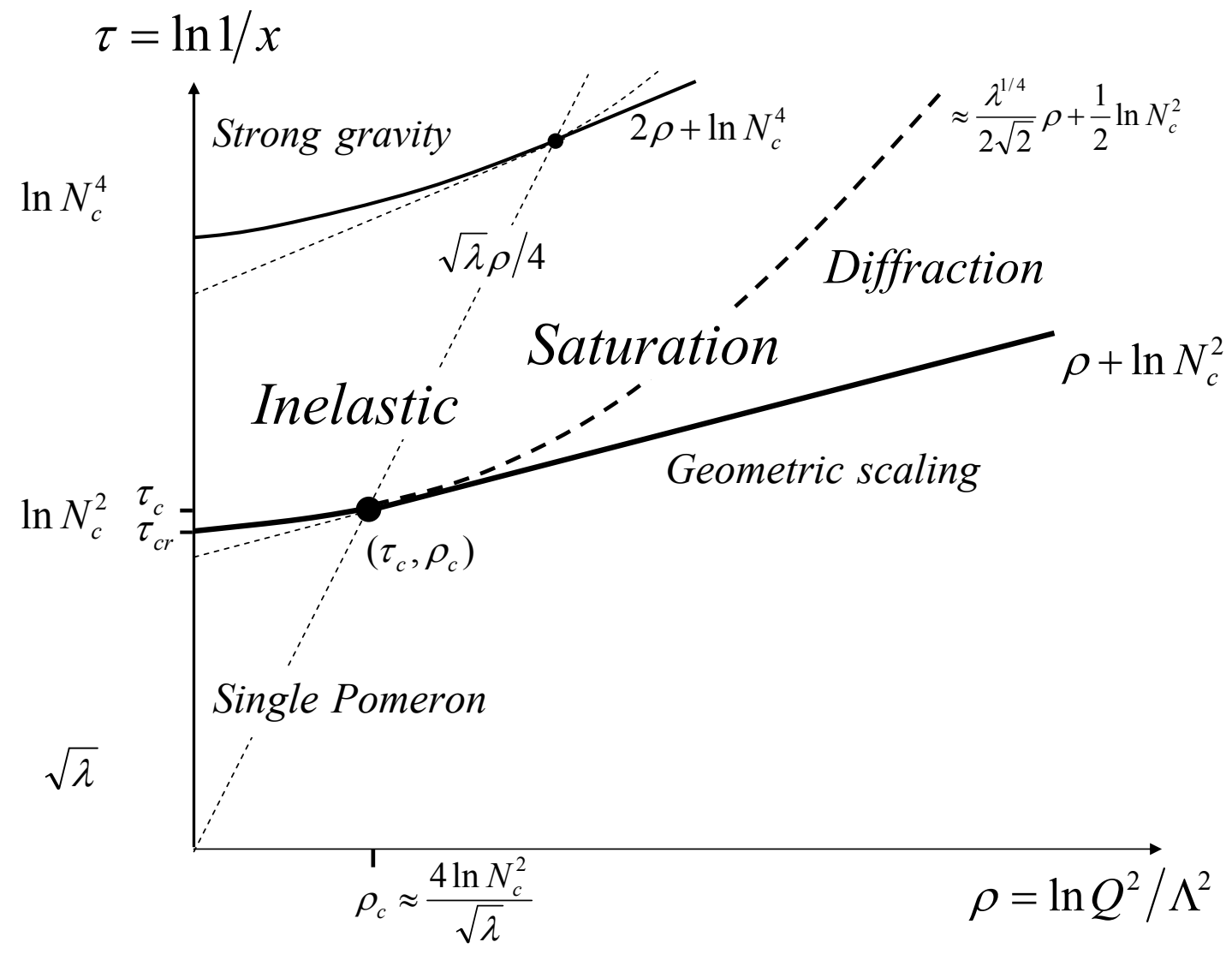

Figure 3: Proposed 'phase diagram' for DIS at high energy and strong coupling when $\ln N_{c}^{2} \gg \sqrt{\lambda}$. The continuous, thick, line represents the saturation line. The other boundaries are explained in the text.

In Fig. 3, the lower branch of the saturation line, Eq. (5.1), is also represented, as a dotted line, for $\tau>\tau_{c}$, where it indicates the 'unitarity limit' for inelastic processes, i.e., the line along which the contribution of the single-Pomeron exchange to the DIS amplitude becomes of $\mathcal{O}(1)$. Note that the 'diffusion approximation' that was implicitly used in deriving the equation of this line in Sect. 3.2 is still valid ${ }^{6}$ for such large values of $\rho$ because, along this line, $j \lesssim 3$, with the upper limit $j=3$ corresponding to $\rho \rightarrow \infty$. (This can be checked by using Eq. (4.25) together with the large $-\rho$ version of Eq. (3.24), that is, $\tau_{s}(\rho) \simeq\left(\lambda^{1 / 4} \rho / \sqrt{2}+\ln N_{c}^{2}\right) / 2$. $)$ Below that line and for $\rho>\rho_{c}$, the DIS cross-section is dominated by the diffractive processes (whose contribution becomes of $\mathcal{O}(1)$ already along the proper saturation curve, Eq. (5.2)), whereas above that line, both diffractive and inelastic processes contribute on equal footing and together give an amplitude $T=1$. Also, below the saturation line (5.2), the DIS cross-section for $\rho>\rho_{c}$ is predominantly diffractive, of higher-twist order, and it exhibits geometric scaling, cf. Eq. (4.40). The upper corner of Fig. 3 denoted as 'strong gravity' will be discussed towards the end of this section.

\footnotetext{
${ }^{6}$ As discussed in Sect. 4.1, the diffusion approximation is expected to be correct so long as $j \ll \sqrt{\lambda}$.
} 
So far we have assumed $N_{c}^{2}>\mathrm{e}^{\sqrt{\lambda}}$, to allow both regimes alluded to above to coexist with each other. But it should be clear that the opposite case $N_{c}^{2} \ll \mathrm{e}^{\sqrt{\lambda}}$ (with $N_{c} \gg 1$, though) is included too in our results, as the special limit $\rho_{c} \rightarrow 0$ : then, the first regime squeezes to zero, meaning that there is not enough phase-space for the genuine Pomeron behaviour to appear the amplitude reaches values of order one via multiple graviton exchanges already before the diffusion becomes important.

Note that the above results do not involve special properties of the dilaton target, like its mass or its conformal dimension $\Delta$. We therefore expect them to universally apply (at strong coupling) to all hadrons which, like the dilaton, have a dual state localized near the infrared cutoff $u_{0}=\Lambda R^{2}$ in the radial dimension of $A d S_{5}$. The same universality applies to the form of the structure function $F_{2}\left(\tau, Q^{2}\right)$ in the vicinity of the saturation line. In the weak-scattering regime at $Q^{2} \gg Q_{s}^{2}(\tau), F_{2}$ shows a rapid rise with $\tau$ and a rapid decrease with $Q^{2}, F_{2} \propto\left(\mathrm{e}^{2 \tau} / Q^{2}\right)$, cf. Eq. (4.39), which is the hallmark of the double-graviton exchange. Furthermore, at low momenta $Q^{2} \lesssim Q_{s}^{2}(\tau)$, one finds $F_{2} \sim N_{c}^{2} Q^{2} / \Lambda^{2}$, cf. Eq. (4.38), a structure which is an almost automatic consequence of the definition (3.15) of $F_{2}$ together with the black disk limit for the scattering amplitude. But in QCD at weak coupling at least, this structure is also tantamount of parton saturation, which makes it tempting to propose a similar interpretation in the strong-coupling case as well.

Let us first briefly recall the situation in pQCD (see, e.g., the review papers [27] for more details): there, the structure function $F_{2}\left(\tau, Q^{2}\right)$ measured in DIS can be interpreted as the quark distribution in the target, as measured in the special frame (the 'infinite momentum frame', or IMF) in which the target has a very large longitudinal momentum $P \gg M$. The 'quark distribution' is the number of quarks per unit rapidity which are localized in impact parameter space within an area $\sim 1 / Q^{2}$ fixed by the resolution of the virtual photon; alternatively — via the uncertainty principle - , this is the number of quark excitations with transverse momenta $k_{\perp} \lesssim Q$. Hence, by knowing $F_{2}\left(\tau, Q^{2}\right)$, one can estimate the quark occupation number as

$$
n_{q}\left(\tau, k_{\perp}\right) \equiv \frac{\mathrm{d} N_{q}}{\mathrm{~d} \tau \mathrm{d}^{2} k_{\perp} \mathrm{d}^{2} b_{\perp}} \simeq \frac{1}{N_{c}} \frac{\mathrm{d} F_{2}}{\mathrm{~d} Q^{2} \pi R_{0}^{2}}
$$

where the derivative in the r.h.s. is evaluated at $Q^{2}=k_{\perp}^{2}, R_{0}$ is the radius of the hadron disk, and the factor $1 / N_{c}$ enters since we consider quarks of a given color. (Other numerical factors, like the number of spin states, or the number of flavors, are not explicitly shown.) Then, by using the estimates (2.30) and (2.31) for $F_{2}$, one can distinguish between two physical regimes: (i) a dilute regime at high momenta $k_{\perp} \gg Q_{s}(\tau)$, where $n_{q}\left(\tau, k_{\perp}\right)$ scales like $n_{q} \sim\left(Q_{s}^{2}(\tau) / k_{\perp}^{2}\right)^{1-\gamma_{s}}$, and thus rises rapidly with $\tau$ due the BFKL evolution, and (ii) a saturation regime at momenta $k_{\perp} \lesssim Q_{s}(\tau)$, where $n_{q}$ saturates at a value of order 1 . This is quark saturation and, as explained in Sect. 2, it ultimately reflects the saturation of the gluon occupation numbers in pQCD at small $x$ [19, 28, 29, 6, 9]: $n_{g}\left(\tau, k_{\perp}\right) \sim 1 / \bar{\alpha}_{s}$ for $k_{\perp} \lesssim Q_{s}(\tau)$.

Returning to the strong-coupling problem of interest, it is clear that a straightforward partonic interpretation is now hindered by the lack of a simple relation between the operators which enter the structure of the $\mathcal{R}$-current (for the $\mathcal{N}=4$ SYM theory, this current receives contributions from the Weyl fermions and from the scalar fields) and the physical excitations in the

\footnotetext{
${ }^{7}$ For very large $k_{\perp}^{2}$, the anomalous dimension dies away, $\gamma_{s} \rightarrow 0$, and one recovers the expected bremsstrahlung spectrum $n_{q} \propto 1 / k_{\perp}^{2}$.
} 
target wavefunction: the latter would be created, or annihilated, by the respective fundamental fields only to lowest order in perturbation theory; but at strong coupling this relation could be strongly renormalized, in a way which is not under control. It is not clear, though, whether such a renormalization will completely wash out the parton picture suggested by perturbation theory. For instance, it is known that the entropy of strongly coupled $\mathcal{N}=4$ SYM theory at finite temperature, which is a direct measure of the number of states, differs only by a factor $3 / 4$ from the entropy of the corresponding ideal gas, where all the states are associated with free fundamental fields. This is suggestive that, at least, the counting of sates is quite similar at strong coupling and at weak coupling.

A similar suggestion emerges from our present results for DIS at strong coupling. When analyzed in view of Eq. (5.3) (with $N_{c} \rightarrow N_{c}^{2}-1 \approx N_{c}^{2}$, to account for the proper number of color degrees of freedom of the fields in $\mathcal{N}=4 \mathrm{SYM}$ ), the estimate (4.38) for $F_{2}$ suggests that, for transverse momenta $k_{\perp} \lesssim Q_{s}(\tau)$, the occupation numbers for the fermionic and scalar fields saturate at a value of order 1 . Note that, for fermions, this value $n=1$ is in agreement with the maximal occupancy allowed by the Pauli principle. Our analysis gives no direct access to the gluon occupancy, but in view of the supersymmetry of the underlying theory, we expect a similar saturation value, $n_{g} \sim 1$ for $k_{\perp} \lesssim Q_{s}(\tau)$, for the gluons as well. This value is quite reasonable: at small coupling, one needs a high occupancy $n_{g} \sim 1 / \bar{\alpha}_{s} \gg 1$ in order to compensate for the weakness of the interactions, but when the coupling is strong, the gluons mutual repulsion responsible for saturation becomes important already when $n_{g} \sim 1$.

By pushing this interpretation towards larger virtualities $Q^{2} \gg Q_{s}^{2}(\tau)$, where Eq. (4.39) applies, one finds that, when increasing $k_{\perp}$ above $Q_{s}(\tau)$, the (scalar and fermion) occupation numbers decrease very fast, as $n \sim\left(Q_{s}^{2}(\tau) / k_{\perp}^{2}\right)^{2}$. Assuming that this behaviour extends to gluons as well, we deduce that, at strong coupling, there are essentially no partons at transverse momenta above the saturation scale: at large $k_{\perp}$, the spectrum converges so fast that any interesting convolution involving the integral of $n\left(\tau, k_{\perp}\right)$ over $k_{\perp}$ will be dominated by $k_{\perp} \lesssim$ $Q_{s}(\tau)$. Equivalently, when the hadron is probed with a given transverse resolution scale $Q^{2}$, almost all partons appear to live at very small values of $x$, or large values of $\tau \equiv \ln (1 / x)$, above the saturation line: $\tau>\tau_{s}\left(Q^{2}\right)$. Since, on the other hand, such small- $x$ partons carry only little longitudinal momentum, it is natural to find that the energy-momentum sum rule is dominated by those partons living along the saturation line, as shown by Eq. (4.37).

It is interesting to notice that the 'phase diagram' in Fig. 3, or, more precisely, a slice of it at fixed $\tau$, bears some resemblance to a previous scenario for high-energy string scattering in flat space by Amati et al [12], after replacing the impact parameter $b$ of Ref. [12] by the photon virtuality $Q^{2}$. Specifically, both pictures have in common the dominance of the diffractive over the inelastic cross-section at large values of $\rho$ (with $\rho=\ln Q^{2}$ in the present context and $\rho=b$ in the case of Ref. [12]) and for sufficiently large values of $\tau$. But a closer inspection reveals also some important differences between these two pictures, which can be attributed to our use of a nontrivial background metric. In particular, and unlike in Ref. [12], in our present analysis there is no hierarchy between the elastic and diffractive processes: they all contribute on the same footing and simultaneously become large on the 'elastic line' in Eq. (4.29).

Consider finally the corner of the 'phase diagram' at ultrahigh energies $s \gtrsim N_{c}^{4}$ which in Fig. 3 is referred to as 'strong gravity'. This is the region where the square of the momentum 
transferred in the $u$ direction, $t=k_{u} k^{u}=G^{u u} k_{u} k_{u}$, which grows with $s$ like $t_{u}(s) \propto s^{2}$ (see the Appendix) becomes of the same order as the total energy squared $s$, meaning that the eikonal approximation cannot be trusted anymore. Physically, this is generally interpreted (so far, mostly in the context of flat space analyses [12, 46, 47]) as the onset of non-linear gravity effects, possibly associated with the formation of a black hole. In this regime, the graviton self-interactions cannot be neglected anymore, so in the context of the scattering problem one has to resum an infinite number of interacting gravitons in the $t$-channel. This is reminiscent of the non-linear, strong gluon field problem of pQCD in the saturation regime [29, 0, 8, 9, 30, 48]. It would be therefore interesting to see if the similarity between the two phenomena could be made even sharper.

\section{Acknowledgments}

We are grateful to R. Brower and C. -I. Tan for answering our questions and providing us with their preliminary results on the eikonal resummation in $A d S_{5}$. We thank I. Bena, L. McLerran, and R. Peschanski for discussions. Y. H. thanks K. Hosomichi, R. Janik, and L. Lipatov for helpful conversations. The work of A. M. is supported in part by the US Department of Energy. The work of E. I. in supported in part by Agence Nationale de la Recherche via the programme ANR-06-BLAN-0285-01.

\section{A. On the breakdown of the eikonal approximation at ultrahigh energies}

In this Appendix, we discuss the breakdown of the eikonal approximation due to large momentum transfer at ultrahigh energies. The momentum $k_{u}$ transferred in the $u$ direction can be estimated as the $u$-derivative of the real part of the phase-shift $\delta$ in the $S$-matrix $\left(S=\mathrm{e}^{i \delta}\right): k_{u} \sim \partial_{u} \delta$.

Consider first the regime at relatively large $Q^{2}$, such that $\rho \equiv \ln \left(Q^{2} / \Lambda^{2}\right)>4 \tau / \sqrt{\lambda}$. Then, as explained in Sects. 4.2 and 4.3 , the phase-shift is controlled by the elementary graviton exchange, which yields

$$
k_{u} \sim \partial_{u}\left(\frac{\mathrm{e}^{\tau}}{N_{c}^{2}} \frac{u_{0}^{2}}{u^{2}}\right) \sim \frac{\mathrm{e}^{\tau}}{N_{c}^{2}} \frac{u_{0}^{2}}{u^{3}},
$$

and hence the invariant squared momentum transfer reads

$$
t \equiv G^{u u} k_{u} k_{u} \sim \frac{1}{R^{2}}\left[\frac{\mathrm{e}^{\tau}}{N_{c}^{2}} \frac{u_{0}^{2}}{u^{2}}\right]^{2},
$$

where we have used $G^{u u}=u^{2} / R^{2}$. When this becomes on the order of the total squared energy $\tilde{s}=\left(R^{2} / u^{2}\right) s$ (the rescaling factor $R^{2} / u^{2}$ is necessary to relate the four dimensional energy to the ten dimensional one [15])

$$
t \sim \frac{R^{2}}{u^{2}} s
$$

the eikonal approximation breaks down. In the context of DIS, this condition must be considered for $u \sim u_{c}=Q R^{2}$, cf. Eq. (3.6), which is the value that controls the respective cross-section. This yields the following critical line

$$
\tau=2 \rho+\ln N_{c}^{4}
$$


above which one expects strong gravity effects, possibly leading to the formation of a black hole.

Consider similarly the low $-Q^{2}$ regime, at $\rho<4 \tau / \sqrt{\lambda}$. Then, the phase shift is associated with the single Pomeron exchange, and can be estimated as in Eq. (4.26) (for both the real and the imaginary part):

$$
\delta \sim \frac{1}{N_{c}^{2}} \mathrm{e}^{\omega_{0} \tau-\frac{\rho^{2}}{4 D \tau}}
$$

The $u$-derivative introduces a factor of order $1 / u\left(\right.$ since $\left.\partial_{u} \rho=2 / u\right)$, so up to subleading prefactors, one finds

$$
t \equiv G^{u u}\left(\partial_{u} \delta\right)^{2} \sim \frac{1}{R^{2} N_{c}^{4}} \mathrm{e}^{2 \omega_{0} \tau-\frac{\rho^{2}}{2 D \tau}}
$$

to be compared with $\tilde{s} \equiv\left(R^{2} / u_{c}^{2}\right) s=\mathrm{e}^{\tau} / R^{2}$. This comparison determines a curve

$$
\left(1-\frac{4}{\sqrt{\lambda}}\right) \tau-\frac{\sqrt{\lambda} \rho^{2}}{4 \tau}=\ln N_{c}^{4},
$$

which smoothly matches the previous line $\tau=2 \rho+\ln N_{c}^{4}$, cf. Eq. (A.4), at a point

$$
(\tau, \rho)=\left(\frac{\ln N_{c}^{4}}{1-8 / \sqrt{\lambda}}, \frac{4 \ln N_{c}^{4}}{\sqrt{\lambda}-8}\right) .
$$

Together, the two lines in Eqs. (A.4) and (A.8) determines the boundary of the applicability of the eikonal approximation at ultrahigh energies $s \gtrsim N_{c}^{4}$, as illustrated in Fig. 3 .

\section{References}

[1] L.N. Lipatov, Reggeization Of The Vector Meson And The Vacuum Singularity In Nonabelian Gauge Theories, Sov. J. Nucl. Phys. 23 (1976) 338;

E.A. Kuraev, L.N. Lipatov and V.S. Fadin, The Pomeranchuk Singularity In Nonabelian Gauge Theories, Zh. Eksp. Teor. Fiz 723 (1977) (Sov. Phys. JETP 45 (1977) 199);

Ya.Ya. Balitsky and L.N. Lipatov, The Pomeranchuk Singularity In Quantum Chromodynamics, Sov. J. Nucl. Phys. 28 (1978) 822.

[2] V. S. Fadin and L. N. Lipatov, BFKL pomeron in the next-to-leading approximation, Phys. Lett. B 429 (1998) 127 [arXiv:hep-ph/9802290;

M. Ciafaloni and G. Camici, Energy scale(s) and next-to-leading BFKL equation, Phys. Lett. B 430 (1998) 349 arXiv:hep-ph/9803389.

[3] G.P. Salam, A resummation of large sub-leading corrections at small x, JHEP 9807 (1998) 19 arXiv:hep-ph/9806482;

M. Ciafaloni, D. Colferai and G. P. Salam, Renormalization group improved small-x equation, Phys. Rev. D 60 (1999) 114036 [arXiv:hep-ph/9905566];

G. Altarelli, R. D. Ball and S. Forte, Perturbatively stable resummed small x evolution kernels, Nucl. Phys. B 742 (2006) 1 arXiv:hep-ph/0512237.

[4] I. Balitsky, Operator expansion for high-energy scattering, Nucl. Phys. B 463 (1996) 99 arXiv:hep-ph/9509348. 
[5] Yu.V. Kovchegov, Small-x F2 structure function of a nucleus including multiple pomeron exchanges, Phys. Rev. D 60 (1999) 034008 arXiv:hep-ph/9901281;

Unitarization of the BFKL pomeron on a nucleus, ibid. D 61 (1999) 074018 arXiv:hep-ph/9905214.

[6] A. H. Mueller, Parton saturation at small $x$ and in large nuclei, Nucl. Phys. B 558 (1999) 285 arXiv:hep-ph/9904404.

[7] J. Jalilian-Marian, A. Kovner, A. Leonidov and H. Weigert, The BFKL equation from the Wilson renormalization group, Nucl. Phys. B 504 (1997) 415 arXiv:hep-ph/9701284; The Wilson renormalization group for low $x$ physics: Towards the high density regime, Phys. Rev. D 59 (1999) 014014 arXiv:hep-ph/9706377;

J. Jalilian-Marian, A. Kovner and H. Weigert, The Wilson renormalization group for low x physics: Gluon evolution at finite parton density, Phys. Rev. D 59 (1999) 014015 arXiv:hep-ph/9709432;

H. Weigert, Unitarity at small Bjorken x, Nucl. Phys. A 703 (2002) 823 arXiv:hep-ph/0004044.

[8] E. Iancu, A. Leonidov and L. McLerran, Nonlinear gluon evolution in the color glass condensate. I, Nucl. Phys. A 692 (2001) 583 arXiv:hep-ph/0011241; The renormalization group equation for the color glass condensate, Phys. Lett. B 510 (2001) 133 arXiv:hep-ph/0102009;

E. Ferreiro, E. Iancu, A. Leonidov and L. McLerran, Nonlinear gluon evolution in the color glass condensate. II, Nucl. Phys. A 703 (2002) 489 arXiv:hep-ph/0109115.

[9] E. Iancu and L. McLerran, Saturation and universality in QCD at small x, Phys. Lett. B 510 (2001) 145 arXiv:hep-ph/0103032.

[10] A. V. Kotikov and L. N. Lipatov, DGLAP and BFKL equations in the $N=4$ supersymmetric gauge theory, Nucl. Phys. B 661 (2003) 19 [Erratum-ibid. B 685 (2004) 405] arXiv:hep-ph/0208220;

J. R. Andersen and A. Sabio Vera, The gluon Green's function in $N=4$ supersymmetric Yang-Mills theory, Nucl. Phys. B 699 (2004) 90 arXiv:hep-th/0406009.

[11] J. M. Maldacena, The large $N$ limit of superconformal field theories and supergravity, Adv. Theor. Math. Phys. 2 (1998) 231 arXiv:hep-th/9711200;

S. S. Gubser, I. R. Klebanov and A. M. Polyakov, Gauge theory correlators from non-critical string theory, Phys. Lett. B 428 (1998) 105 arXiv:hep-th/9802109;

E. Witten, Anti-de Sitter space and holography, Adv. Theor. Math. Phys. 2 (1998) 253 arXiv:hep-th/9802150.

[12] D. Amati, M. Ciafaloni and G. Veneziano, Superstring Collisions at Planckian Energies, Phys. Lett. B 197 (1987) 81; Classical and Quantum Gravity Effects from Planckian Energy Superstring Collisions, Int. J. Mod. Phys. A 3 (1988) 1615; Can Space-Time Be Probed Below The String Size?, Phys. Lett. B 216 (1989) 41.

[13] R. A. Janik and R. Peschanski, High energy scattering and the AdS/CFT correspondence, Nucl. Phys. B 565 (2000) 193 arXiv:hep-th/9907177]; Minimal surfaces and Reggeization in the AdS/CFT correspondence, ibid. B 586 (2000) 163 [arXiv:hep-th/0003059];

S. B. Giddings, High energy QCD scattering, the shape of gravity on an IR brane, and the Froissart bound, Phys. Rev. D 67 (2003) 126001 arXiv:hep-th/0203004;

O. Andreev and W. Siegel, Quantized tension: Stringy amplitudes with Regge poles and parton behavior, Phys. Rev. D 71 (2005) 086001 arXiv:hep-th/0410131;

K. Kang and H. Nastase, High energy QCD from Planckian scattering in AdS and the Froissart bound, Phys. Rev. D 72 (2005) 106003 arXiv:hep-th/0410173; 
L. Alvarez-Gaume, C. Gomez and M. A. Vazquez-Mozo, Scaling Phenomena in Gravity from QCD, Phys. Lett. B 649 (2007) 478 arXiv:hep-th/0611312;

A. M. Stasto, The BFKL Pomeron in the weak and strong coupling limits and kinematical constraints, Phys. Rev. D 75 (2007) 054023 arXiv:hep-ph/0702195;

L. F. Alday and J. M. Maldacena, Gluon scattering amplitudes at strong coupling, JHEP $\mathbf{0 7 0 6}$ (2007) 064 arXiv:0705.0303 [hep-th]].

[14] J. Polchinski and M. J. Strassler, Hard scattering and gauge/string duality, Phys. Rev. Lett. 88 (2002) 031601 arXiv:hep-th/0109174.

[15] J. Polchinski and M. J. Strassler, Deep inelastic scattering and gauge/string duality, JHEP 0305 (2003) 012 arXiv:hep-th/0209211.

[16] R. C. Brower, J. Polchinski, M. J. Strassler and C. I. Tan, The pomeron and gauge / string duality, arXiv:hep-th/0603115.

[17] L. Cornalba, M. S. Costa, J. Penedones and R. Schiappa, Eikonal approximation in AdS/CFT: From shock waves to four-point functions, arXiv:hep-th/0611122;

L. Cornalba, M. S. Costa and J. Penedones, Eikonal Approximation in AdS/CFT: Resumming the Gravitational Loop Expansion, arXiv:0707.0120 [hep-th]].

[18] R. C. Brower, M. J. Strassler and C. I. Tan, On the Eikonal Approximation in AdS Space, [arXiv:0707.2408[hep-th];

R. C. Brower and C. -I. Tan, talks given at The 9-th workshop on non-perturbative QCD, Paris, June 4-8 (2007).

[19] L.V. Gribov, E.M. Levin, and M.G. Ryskin, Semihard Processes In QCD, Phys. Rept. 100 (1983) 1.

[20] E. Iancu, K. Itakura, and L. McLerran, Geometric scaling above the saturation scale, Nucl. Phys. A 708 (2002) 327 arXiv:hep-ph/0203137.

[21] A.H. Mueller and D.N. Triantafyllopoulos, The energy dependence of the saturation momentum, Nucl. Phys. B 640 (2002) 331 arXiv:hep-ph/0205167.

[22] S. Munier and R. Peschanski, Geometric scaling as traveling waves, Phys. Rev. Lett. 91 (2003) 232001 arXiv:hep-ph/0309177.

[23] J. Polchinski, String theory. Vol. 1: An introduction to the bosonic string, Cambridge, UK: Univ. Pr. (1998) 402 p; String theory. Vol. 2: Superstring theory and beyond, Cambridge, UK: Univ. Pr. (1998) $531 \mathrm{p}$.

[24] N.N. Nikolaev and B.G. Zakharov, Colour transparency and scaling properties of nuclear shadowing in deep inelastic scattering, Z. Phys. C 49 (1991) 607.

[25] A.H. Mueller, Soft Gluons In The Infinite Momentum Wave Function And The Bfkl Pomeron, Nucl. Phys. B 415 (1994) 373;

A.H. Mueller and B. Patel, Single And Double Bfkl Pomeron Exchange And A Dipole Picture Of High-Energy Hard Processes, ibid. B 425 (1994) arXiv:hep-ph/9403256;

A.H. Mueller, Unitarity and the BFKL pomeron, ibid. B 437 (1995) 107 arXiv:hep-ph/9408245.

[26] J. R. Forshaw and D. A. Ross, Quantum Chromodynamics and the Pomeron, Cambridge University Press, Cambridge, 1997. 
[27] A. H. Mueller, Parton Saturation-An Overview, arXiv:hep-ph/0111244, in QCD Perspectives on Hot and Dense Matter, Eds. J.-P. Blaizot and E. Iancu, NATO Science Series, Kluwer, 2002;

E. Iancu and R. Venugopalan, The Color Glass Condensate and High Energy Scattering in QCD, arXiv:hep-ph/0303204, in Quark-Gluon Plasma 3, Eds. R. C. Hwa et al, World Scientific, 2003.

[28] A.H. Mueller and J. Qiu, Gluon Recombination And Shadowing At Small Values Of X, Nucl. Phys. B 268 (1986) 427.

[29] L. McLerran and R. Venugopalan, Computing quark and gluon distribution functions for very large nuclei, Phys. Rev. D 49 (1994) 2233 arXiv:hep-ph/9309289]; Gluon distribution functions for very large nuclei at small transverse momentum, ibid. D 49 (1994) 3352 arXiv:hep-ph/9311205.

[30] E. Iancu and D.N. Triantafyllopoulos, A Langevin equation for high energy evolution with pomeron loops, Nucl. Phys. A 756 (2005) 419 [arXiv:hep-ph/0411405]; Non-linear QCD evolution with improved triple-pomeron vertices, Phys. Lett. B 610 (2005) 253 arXiv:hep-ph/0501193;

A.H. Mueller, A.I. Shoshi and S.M.H. Wong, Extension of the JIMWLK equation in the low gluon density region, Nucl. Phys. B 715 (2005) 440 arXiv:hep-ph/0501088.

[31] D.N. Triantafyllopoulos, The energy dependence of the saturation momentum from RG improved BFKL evolution, Nucl. Phys. B 648 (2003) 293 arXiv:hep-ph/0209121.

[32] S. Munier and R. Peschanski, Traveling wave fronts and the transition to saturation, Phys. Rev. D 69 (2004) 034008 arXiv:hep-ph/0310357;

V. A. Khoze, A. D. Martin, M. G. Ryskin and W. J. Stirling, The spread of the gluon $k(t)$-distribution and the determination of the saturation scale at hadron colliders in resummed NLL BFKL, Phys. Rev. D 70074013 (2004) arXiv:hep-ph/0406135;

I. Balitsky, Quark contribution to the small-x evolution of color dipole, Phys. Rev. D 75 (2007) 014001 arXiv:hep-ph/0609105;

Yu.V. Kovchegov and H. Weigert, Triumvirate of running couplings in small-x evolution, Nucl. Phys. A 784 (2007) 188 [arXiv:hep-ph/0609090]; Quark loop contribution to BFKL evolution: Running coupling and leading-N(f) NLO intercept Nucl. Phys. A 789 (2007) 260 arXiv:hep-ph/0612071;

J.L. Albacete and Yu.V. Kovchegov, Solving High Energy Evolution Equation Including Running Coupling Corrections, arXiv:0704.0612 [hep-ph].

[33] J. C. Collins and R. K. Ellis, Heavy quark production in very high-energy hadron collisions, Nucl. Phys. B 360 (1991) 3;

S. Catani, M. Ciafaloni and F. Hautmann, High-energy factorization and small $x$ heavy flavor production, Nucl. Phys. B 366 (1991) 135.

[34] K. Golec-Biernat and M. Wüsthoff, Saturation effects in deep inelastic scattering at low $Q^{* * 2}$ and its implications on diffraction, Phys. Rev. D 59 (1999) 014017 arXiv:hep-ph/9807513];

Saturation in diffractive deep inelastic scattering, ibid. D 60 (1999) 114023 arXiv:hep-ph/9903358.

[35] A.M. Stasto, K. Golec-Biernat and J. Kwiecinski, Geometric scaling for the total gamma* $p$ cross-section in the low $x$ region, Phys. Rev. Lett. 86 (2001) 596 arXiv:hep-ph/0007192.

[36] E. Iancu, K. Itakura and S. Munier, Saturation and BFKL dynamics in the HERA data at small $x$, Phys. Lett. B 590 (2004) 199 arXiv:hep-ph/0310338.

[37] T. Jaroszewicz, Gluonic Regge Singularities And Anomalous Dimensions In QCD, Phys. Lett. B 116291 (1982);

L. N. Lipatov, The Bare Pomeron In Quantum Chromodynamics, Sov. Phys. JETP 63 (1986) 904. 
[38] A. V. Kotikov, L. N. Lipatov, A. I. Onishchenko and V. N. Velizhanin, Three-loop universal anomalous dimension of the Wilson operators in $N=4$ SUSY Yang-Mills model, Phys. Lett. B 595 (2004) 521 [Erratum-ibid. B 632 (2006) 754] arXiv:hep-th/0404092].

[39] S. S. Gubser, I. R. Klebanov and A. M. Polyakov, A semi-classical limit of the gauge/string correspondence, Nucl. Phys. B 636 (2002) 99 arXiv:hep-th/0204051.

[40] V. N. Gribov, The theory of complex angular momenta: Gribov lectures on theoretical physics, Cambridge, UK: Univ. Pr. (2003) 297 p.

[41] A. A. Tseytlin, On semiclassical approximation and spinning string vertex operators in AdS(5) $x$ S(5), Nucl. Phys. B 664 (2003) 247 arXiv:hep-th/0304139.

[42] C. G. Callan and Z. Gan, Vertex Operators In Background Fields, Nucl. Phys. B 272 (1986) 647.

[43] G. 't Hooft, Graviton Dominance in Ultrahigh-Energy Scattering, Phys. Lett. B 198 (1987) 61.

[44] Y. Hatta and A. H. Mueller, Correlation of small-x gluons in impact parameter space, Nucl. Phys. A 789 (2007) 285 arXiv:hep-ph/0702023.

[45] S. B. Giddings, Locality in quantum gravity and string theory, Phys. Rev. D 74 (2006) 106006 arXiv:hep-th/0604072.

[46] G. Veneziano, String-theoretic unitary S-matrix at the threshold of black-hole production, JHEP 0411 (2004) 001 arXiv:hep-th/0410166.

[47] S. B. Giddings, D. J. Gross and A. Maharana, Gravitational effects in ultrahigh-energy string scattering, arXiv:0705.1816 [hep-th].

[48] I. Balitsky, Factorization and high-energy effective action, Phys. Rev. D 60 (1999) 014020 arXiv:hep-ph/9812311;

Y. Hatta, E. Iancu, L. McLerran, A. Stasto and D. N. Triantafyllopoulos, Effective Hamiltonian for QCD evolution at high energy, Nucl. Phys. A 764 (2006) 423 arXiv:hep-ph/0504182.

[49] Y. Hatta, E. Iancu, C. Marquet, G. Soyez, D.N. Triantafyllopoulos, Diffusive scaling and the high-energy limit of deep inelastic scattering in QCD at large N(c), Nucl. Phys. A 773 (2006) 95 arXiv:hep-ph/0601150. 